\title{
Evaluación perceptual de la insuficiencia velofaríngea en personas con fisura del paladar: una revisión integradora de literatura
}

\author{
Felipe Inostroza-Allende a, b, *, Gustavo Baeza-Pavez, Paula Del Valle-Román, Jason Fernández-Antifil, \\ Constanza Yáñez-Pavez a , Josué Pino-Castillo a \\ a Departamento de Fonoaudiología, Universidad de Chile, Chile \\ b Fundación Gantz, Pudahuel, Chile
}

\section{RESUMEN}

La insuficiencia velofaríngea (IVF) secundaria de fisura del paladar corresponde al cierre incompleto del mecanismo velofaríngeo durante el habla, debido a una falta de tejido en el paladar blando o las paredes de la faringe, lo cual genera una resonancia hipernasal y una emisión nasal de aire en los sonidos orales. Al respecto, en la literatura existen diversas propuestas para la evaluación perceptual de la IVF. Por esto, el objetivo del presente estudio es describir la evaluación perceptiva auditiva de la insuficiencia velofaríngea, mediante una revisión integradora de literatura. Para ello, en mayo de 2020 las bases de datos electrónicas PUBMED, LILACS, SciELO y Cochrane, fueron consultadas utilizando las palabras claves en inglés: "Velopharyngeal Sphincter", "Velopharyngeal Insufficiency", "Cleft Palate", "Speech Intelligibility", "Speech Production Measurement", "Speech Articulation Tests" y "Speech-Language Pathology" y sus respectivos equivalentes en portugués y español. Se seleccionaron artículos originales relacionados al tema, y se creó un protocolo específico para la extracción de los datos. En total se encontraron 2.385 artículos. De ellos, 2.354 fueron excluidos por el título, 13 por el resumen y 3 luego de la lectura del texto completo. Finalmente, a partir de la metodología desarrollada, en esta revisión fueron utilizados 33 artículos. A partir de la revisión realizada se concluye que los parámetros más utilizados en la evaluación son la hipernasalidad, la emisión nasal y la articulación compensatoria asociada a IVF. Estos parámetros son evaluados principalmente en oraciones, habla espontánea y palabras, por un fonoaudiólogo experto, en vivo y mediante grabaciones de audio.

\section{Perceptual evaluation of velopharyngeal insufficiency in people with cleft palate: an integrative literature review}

\section{ABSTRACT}

Secondary Velopharyngeal insufficiency (VPI) to cleft palate corresponds to the incomplete closure of the velopharyngeal mechanism during speech, due to lack of tissue in the soft palate or the walls of the pharynx, which generates a hypernasal resonance and nasal air emission in oral sounds. In this regard, there are various proposals in the literature for the perceptual evaluation of VPI. For this reason, the objective of the present study is to describe the auditory perceptual evaluation of velopharyngeal insufficiency, through an integrative literature review. To this end, in May 2020 the electronic databases PUBMED, LILACS, SciELO and Cochrane were consulted using the key words in English: "Velopharyngeal Sphincter", "Velopharyngeal Insufficiency", "Cleft Palate", "Speech Intelligibility", "Speech Production Measurement", "Speech Articulation Tests" and "Speech-Language Pathology", and their equivalent properties in Portuguese and Spanish. Original articles related to the topic were selected, and a specific protocol for data extraction was created. In total, 2,385 articles were found. Of these, 2,354 were excluded due to the title, 13 due to the abstract and 3 after reading the full text. Finally, based on the methodology developed, 33 articles were used in this review. From the review carried out, it is concluded that the parameters most used in the evaluation are hypernasality, nasal emission and the compensatory joint associated with IVF. These parameters are evaluated mainly in sentences, spontaneous speech and words, by an expert speech therapist, live and through audio recordings.

*Autor/a correspondiente: Felipe Inostroza-Allende

Email:f.inostrozarp@gmail.com

\section{Keywords: \\ Velopharyngeal Insufficiency; Cleft Palate; Speech-Language Pathology; Speech Production Measurement}




\section{INTRODUCCIÓN}

El mecanismo velofaríngeo (MVF) o esfínter velofaríngeo se encarga de controlar el equilibrio de resonancia entre la cavidad nasal y oral, controlando así las presiones aéreas y acústicas del habla. De esta manera, el esfínter velofaríngeo permanece cerrado durante la producción de los sonidos orales y abierto en los sonidos nasales (Marrinan \& Shprintzer, 2006; Moon, 2004; Smith \& Kuehn, 2007). Cuando el cierre velofaríngeo no ocurre de forma apropiada durante la emisión de sonidos orales, parte de la corriente aérea sonorizada se desvía hacia la cavidad nasal, comprometiendo de diferentes formas la producción del habla (Kuehn \& Moller, 2000). Es así como el exceso de energía acústica en la cavidad nasal altera el equilibrio de la resonancia y acústica del habla (Lam et al., 2007; Smith \& Guyette, 2004).

La insuficiencia velofaríngea (IVF) es el término empleado para referirse al cierre inapropiado del MVF durante el habla, la que puede deberse a alteraciones anatómicas velofaríngeas causadas por cualquier déficit estructural del velo o las paredes de la faringe donde no existe tejido suficiente que asegure un cierre eficiente del MVF. Una causa frecuente de la IVF son las fisuras orofaciales que afectan el paladar (Morris \& Ozanne, 2003; TrostCardamone, 1989). La fisura del paladar es una malformación congénita que se produce por una fusión incompleta o ausente de los procesos maxilares durante la embriogénesis. En la fisura velopalatina se compromete el paladar óseo y blando; en la fisura velar se afectan los músculos y mucosa del paladar blando; y en la fisura submucosa existe una alteración en la musculatura velar, pero con continuidad de la mucosa oral y nasal (Tresserra et al., 1997).

En la fisuras con compromiso del paladar, la corrección quirúrgica primaria del paladar prioriza el establecimiento de condiciones anatómicas y funcionales para un cierre velofaríngeo adecuado (Agrawal, 2009; Becker et al., 2000; Henningsson et al., 2008; Rosanowski \& Eysholdt, 2002). Sin embargo, cerca del 5\% al $36 \%$ de los pacientes con fisura del paladar permanecen con síntomas de IVF luego de la cirugía primaria (Bicknell et al., 2002; Marrinan et al., 1998; Sommerlad, 2003).

La evaluación de la IVF se realiza mediante una evaluación instrumental y una evaluación clínica fonoaudiológica (Ministerio de Salud [MINSAL], 2015). En cuanto a los procedimientos instrumentales, estos pueden ser utilizados tanto en evaluaciones directas como indirectas. Dentro de los que evalúan el funcionamiento del EVF de forma directa, podemos mencionar la videonasofaringoscopia flexible (VNF) y la videofluoroscopia multiplano (VFC). Por otro lado, dentro de la evaluación indirecta, la más utilizada internacionalmente es la nasometría (Bettens et al., 2016; Conley et al., 1997). En tanto, la evaluación clínica perceptual de la IVF realizada por fonoaudiólogos es considerada el procedimiento gold standard para poder evaluar las alteraciones funcionales del MVF durante el habla (Chapman et al., 2016; Kuehn \& Moller, 2000; Smith \& Guyette, 2004).

Debido a lo anteriormente expuesto, es relevante conocer los diversos parámetros y procedimientos descritos para la evaluación perceptual de la función velofaríngea durante el habla luego de intervenciones quirúrgicas, protésicas y funcionales. Actualmente, los parámetros más utilizados son los llamados parámetros universales descritos por Henningsson et al. (2008). Estos parámetros fueron elaborados en un taller realizado en Washington, DC, donde un grupo de trabajo de seis personas con experiencia en habla y fisura del paladar desarrollaron un sistema de parámetros universales para informar los resultados del habla en personas que nacen con fisura del paladar (Henningsson et al., 2008). Sin embargo, existen algunos estudios que describen otros parámetros y escalas de puntuación para la evaluación fonoaudiológica de la IVF, que son utilizadas a nivel nacional e internacional por equipos de rehabilitación multidisciplinaria de personas con fisura del paladar (Álvarez et al., 2004; Henningsson et al., 2008; Kummer, 2011; MINSAL, 2015).

En los últimos años ha ocurrido un creciente desarrollo de diversos tipos de revisiones de literatura que permiten entregar una síntesis de conocimientos sobre una determinada temática. Entre estas, la revisión integradora de literatura es un método que sintetiza investigaciones enfocadas en la práctica clínica, para una posible repercusión benéfica en la calidad de los servicios entregados a los pacientes (Souza et al., 2010). Diversas revisiones integradoras han sido realizadas en aspectos de evaluación e intervención fonoaudiológica, pero aún no se han realizado este tipo de revisiones relacionadas a la evaluación perceptual de la IVF. De esta manera, se propone una revisión integradora de literatura con el objetivo de describir los parámetros y procedimientos del análisis perceptivo auditivo utilizados para la evaluación de la insuficiencia velofaríngea de personas con fisura del paladar operada.

\section{MATERIAL Y MÉTODO}

En la presente revisión fueron ejecutadas las siguientes etapas: 1) Establecimiento de la pregunta de investigación. 2) Búsqueda o muestreo en la literatura. 3) Recolección de datos. 4) Evaluación y análisis crítico de los estudios incluidos. 5) Interpretación y discusión de los resultados. 6) Síntesis del conocimiento y 
presentación de la revisión (Mendes et al., 2008; Souza et al., 2010).

\section{Estrategia de búsqueda}

La elaboración de la revisión se basó en la pregunta: ¿qué parámetros perceptuales y procedimientos se consideran en la evaluación fonoaudiológica de la insuficiencia velofaríngea secundaria a fisura del paladar?

En mayo de 2020 se realizó una búsqueda electrónica sin límite de fecha de publicación, en las siguientes bases de datos: US
National Library of Medicine National Institutes of Health (PUBMED), Literatura Latino-Americana e do Caribe em Ciências da Saúde (LILACS), Scientific Electronic Library Online (SciELO) y Biblioteca Cochrane.

Para la búsqueda de los artículos se utilizaron términos en inglés, disponibles en el Medical Subject Headings (MeSH), y sus equivalentes en español y portugués obtenidos en los Descriptores en Ciencias de la Salud (DeCS). Los términos fueron combinados entre sí con la utilización del operador booleano AND (Tabla 1). Además, se realizó un análisis de referencias cruzadas.

Tabla 1. Descriptores combinados en la búsqueda electrónica.

\begin{tabular}{|c|c|c|}
\hline Inglés (MeSH) & Español (DeCS) & Portugués (DeCS) \\
\hline Velopharyngeal Sphincter AND Speech & Esfínter Velofaríngeo AND Inteligibilidad del & Esfíncter Velofaríngeo AND Inteligibilidade da \\
\hline Intelligibility & Habla & Fala \\
\hline Velopharyngeal Sphincter AND Speech & Esfínter Velofaríngeo AND Medición de la & Esfincter Velofaríngeo AND Medida da \\
\hline Production Measurement & Producción del Habla & Produção da Fala \\
\hline Velopharyngeal Sphincter AND Speech & Esfínter Velofaríngeo AND Pruebas de & Esfïncter Velofaríngeo AND Testes de \\
\hline Articulation Tests & Articulación del Habla & Articulação da Fala \\
\hline Velopharyngeal Sphincter AND Speech- & Esfinter Velofaríngeo AND Patología del & Esfíncter Velofaríngeo AND Patologia da Fala \\
\hline Language Pathology & Habla y Lenguaje & e Linguagem \\
\hline Velopharyngeal Insufficiency AND Speech & Insuficiencia Velofaríngea AND Inteligibilidad & Insuficiência Velofaríngea AND \\
\hline Intelligibility & del Habla & Inteligibilidade da Fala \\
\hline Velopharyngeal Insufficiency AND Speech & Insuficiencia Velofaríngea AND Medición de & Insuficiência Velofaríngea AND Medida da \\
\hline production measurement & la Producción del Habla & Produção da Fala \\
\hline Velopharyngeal Insufficiency AND Speech & Insuficiencia Velofaríngea AND Pruebas de & Insuficiência Velofaríngea AND Testes de \\
\hline Articulation Tests & Articulación del Habla & Articulação da Fala \\
\hline Velopharyngeal Insufficiency AND Speech- & Insuficiencia Velofaríngea AND Patología del & Insuficiência Velofaríngea AND Patologia da \\
\hline Language Pathology & Habla y Lenguaje & Fala e Linguagem \\
\hline Cleft Palate AND Speech Intelligibility & $\begin{array}{l}\text { Fisura del Paladar AND Inteligibilidad del } \\
\text { Habla }\end{array}$ & Fissura Palatina AND Inteligibilidade da Fala \\
\hline $\begin{array}{l}\text { Cleft Palate AND Speech production } \\
\text { measurement }\end{array}$ & $\begin{array}{l}\text { Fisura del Paladar AND Medición de la } \\
\text { Producción del Habla }\end{array}$ & $\begin{array}{l}\text { Fissura Palatina AND Medida da Produção da } \\
\text { Fala }\end{array}$ \\
\hline Cleft Palate AND Speech Articulation Tests & $\begin{array}{l}\text { Fisura del Paladar AND Pruebas de } \\
\text { Articulación del Habla }\end{array}$ & $\begin{array}{l}\text { Fissura Palatina AND Testes de Articulação da } \\
\text { Fala }\end{array}$ \\
\hline Cleft Palate AND Speech-Language Pathology & $\begin{array}{l}\text { Fisura del Paladar AND Patología del Habla y } \\
\text { Lenguaje }\end{array}$ & $\begin{array}{l}\text { Fissura Palatina AND Patologia da Fala e } \\
\text { Linguagem }\end{array}$ \\
\hline
\end{tabular}

\section{Criterios de selección y análisis de los datos}

Los criterios de inclusión definidos para la selección de los estudios fueron: a) Publicados en idiomas inglés, español y portugués. b) Con acceso al texto completo. c) Realizados en personas con fisura del paladar operada. d) Que reportan y describen parámetros de evaluación perceptual de la insuficiencia velofaríngea. e) Con alguno de los siguientes tipos de estudio: serie de casos, estudio de cohorte, ensayo clínico sin asignación aleatoria y aleatorizados.

Para la selección de los estudios, se realizó una lectura de los títulos y resúmenes de las publicaciones localizadas por la estrategia de búsqueda. En seguida, se procedió a la lectura de los artículos seleccionados, que cumplieron los criterios de inclusión y que permitieron responder a la pregunta de esta investigación. 
Cada artículo fue evaluado por dos de los autores y, en caso de duda, hubo consenso entre todos los autores.

Se excluyeron reportes de caso, revisiones de literatura, presentaciones en congresos, tesis y capítulos de libro. Además, fueron excluidos estudios realizados en personas con insuficiencia velofaríngea adquirida por trauma o resección tumoral, incompetencia velofaríngea por lesión neurológica o trastorno del aprendizaje velofaríngeo por déficit auditivo.

Para la extracción de los datos fue diseñado un protocolo que contempla los siguientes puntos: autor, año, país, características de los sujetos con fisura del paladar (número, edad, tipo de fisura, edad del cierre de paladar, fístulas y presencia de articulación compensatoria), características del grupo control (número y edad), objetivo del estudio, parámetros perceptuales para evaluar IVF, muestra de habla para evaluar IVF (espontánea, automática, recontado, sílabas, palabras, oraciones, etc.), evaluador (terapeuta experto; terapeuta no experto; oyente no entrenado), escala (intervalo de apariencia igual - IAI, estimación de magnitud directa - EMD o escala visual analógica - EVA), análisis (en vivo, audio, video), evaluación instrumental (videonasofaringoscopia, videofluoroscopia, nasometría), y principales resultados.

Los resultados serán presentados en formato de tablas. En tanto, los principales hallazgos serán visualizados en gráficos de barras, diseñados en el software jamovi, versión 1.1 (The jamovi project, 2019).

Tabla 2. Resumen de la identificación (año y país) y los participantes de los estudios.

\begin{tabular}{|c|c|c|c|c|c|c|c|}
\hline \multirow[t]{2}{*}{ Autores (año) } & \multirow[t]{2}{*}{ País } & \multicolumn{4}{|c|}{ Grupo de participantes con fisura del paladar } & \multicolumn{2}{|c|}{ Grupo control } \\
\hline & & $\mathrm{N}$ & edad & $\mathrm{Cx} 1^{\circ}($ edad $)$ & Fístulas (sí, no) & $\mathrm{N}$ & edad \\
\hline Nellis et al. (1992) & EEUU & 16 & $8-18 \mathrm{a}$ & NR & NR & NR & NR \\
\hline Williams et al. (1998) & Rusia & 112 & $4-10 \mathrm{a}$ & $2-4 \mathrm{a}$ & sí & NR & NR \\
\hline Keuning et al. (1999) & NL & 15 & $10-13$ a & NR & no & NR & NR \\
\hline Sell et al. (2001) & UK & 647 & $5-12$ a & NR & NR & NR & NR \\
\hline Keuning et al. (2002) & NL & 43 & $4-83$ a & NR & NR & $\mathrm{NE}$ & NE \\
\hline Konst et al. (2003) & Holanda & 54 & $2.5-3 a$ & $12 \mathrm{~m}$ & NR & 8 & 2 \\
\hline Kummer et al. (2003) & EEUU & 173 & $3-12 \mathrm{a}$ & NR & NR & NR & NR \\
\hline Lewis et al. (2003) & EEUU & 17 & 4.2 y $18.4 \mathrm{a}$ & NR & NR & 3 & $4.2-18.4 \mathrm{a}$ \\
\hline Álvarez et al. (2004) & Chile & 46 & 3-29a & NR & NR & NR & NR \\
\hline Keuning et al. (2004) & Holanda & 43 & $4-83 \mathrm{a}$ & NR & NR & NR & NR \\
\hline Paal et al. (2005) & Alemania & 12 & $9.5 \pm 0.5 \mathrm{a}$ & NR & NR & NR & NR \\
\hline John et al. (2006) & UK & 10 & $5-10$ a & NR & NR & 1 & $\mathrm{NE}$ \\
\hline Sweeney \& Sell (2008) & Irlanda & 50 & $4.10-15.10 \mathrm{a}$ & NR & NR & NR & NR \\
\hline
\end{tabular}

\section{RESULTADOS}

En la revisión fue encontrado un total de 2.385 artículos, de los cuales 2.354 fueron excluidos luego de la lectura del título, 13 luego de la lectura de los resúmenes y 3 tras la lectura de los textos completos. Según los criterios metodológicos establecidos previamente y el análisis de referencias cruzadas, 33 artículos fueron analizados en esta revisión (Figura 1).

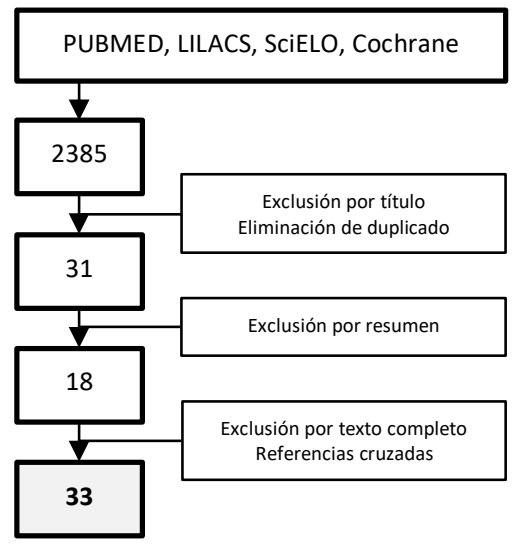

Figura 1. Etapas del proceso de revisión de literatura.

La Tabla 2 y Tabla 3 resumen los estudios seleccionados para la descripción de los parámetros perceptuales y procedimientos utilizados en la evaluación de la insuficiencia velofaríngea de personas con fisura del paladar operada. 


\begin{tabular}{|c|c|c|c|c|c|c|c|}
\hline Lee et al. (2009) & Irlanda & 20 & $21-65 a$ & NR & NR & 2 & $23-35 a$ \\
\hline Rullo et al. (2009) & Italia & 68 & $5-8 \mathrm{a}$ & $8-12 \mathrm{~m}$ & NR & NR & NR \\
\hline Lipira et al. (2011) & EEUU & 88 & $2-24$ a & NR & NR & NR & NR \\
\hline Hubbard et al. (2013) & EEUU & 18 & $3-19 a$ & $3-12 \mathrm{~m}$ & NR & NR & NR \\
\hline Paniagua et al. (2013) & Brasil & 49 & $9-16$ a & $19.5 \mathrm{~m}$ & NR & NR & NR \\
\hline Trindade et al. (2014) & Brasil & 20 & $17-35$ a & NR & NR & 18 & $20-35$ a \\
\hline Nguyen et al. (2015) & EEUU & 249 & 3:00 a. m. & $14-15 \mathrm{~m}$ & NR & NR & NR \\
\hline Padilha et al. (2015) & Brasil & 100 & 5-12 a & $9-18 \mathrm{~m}$ & NR & NR & NR \\
\hline Scarmagnani et al. (2015) & Brasil & 100 & $6-47 \mathrm{a}$ & NR & NR & NR & NR \\
\hline Sell et al. (2015) & UK & 248 & 5 y $12 \mathrm{a}$ & NR & sí & NR & NR \\
\hline Bettens et al. (2016) & Bélgica & 35 & $4-15$ a & NR & NR & NR & NR \\
\hline Chapman et al. (2016) & EEUU & 10 & $5-7$ a & NR & NR & NR & NR \\
\hline Georgievska et al. (2016) & Macedonia & 10 & $4-7 \mathrm{a}$ & NR & NR & 10 & $4-7 \mathrm{a}$ \\
\hline Larangeira et al. (2016) & Brasil & 331 & $5-13$ a & NR & NR & NR & NR \\
\hline Medeiros et al. (2016) & Brasil & 60 & $6-52 \mathrm{a}$ & NR & NR & NR & NR \\
\hline Oliveira et al. (2016) & Brasil & 77 & NR & NR & NR & NR & NR \\
\hline Sinko et al. (2017) & Austria & 36 & $8-27$ a & NR & NR & NR & NR \\
\hline Abdali \& Yaribakht (2019) & Irán & 24 & $13-41 \mathrm{a}$ & $6 \mathrm{~m}$ & NR & NR & NR \\
\hline Aparna et al. (2019) & India & 25 & $5-7 \mathrm{a}$ & $18 \mathrm{~m}$ & NR & NR & NR \\
\hline de Boer et al. (2020) & Canadá & 54 & NR & NR & NR & NR & $7.7 \pm 1.1 \mathrm{a}$ \\
\hline
\end{tabular}

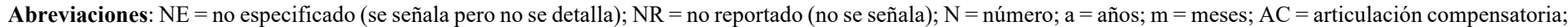
$\mathrm{Cx} 1^{\circ}=$ edad cirugía primaria de paladar; $\mathrm{NL}=$ Países Bajos; $\mathrm{UK}=$ Reino Unido; EEUU $=$ Estados Unidos

En relación a los años de publicación, el primer artículo incluido corresponde a la investigación de Nellis, publicada el año 1992. Al respecto, como se observa en la figura 2, el periodo con mayor producción científica ocurre entre los años 2003 y 2019. En cuanto a los países de publicación, como se observa en la figura 3, los tres países con mayor producción científica en el área son: Brasil, EEUU y UK.

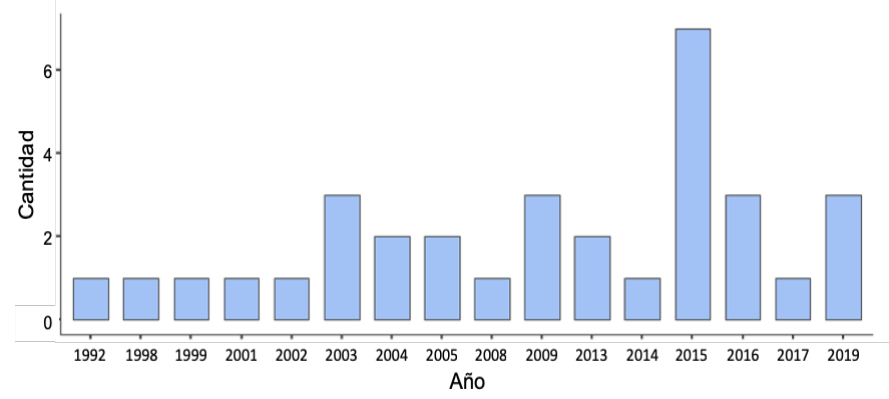

Figura 2. Años de publicación.

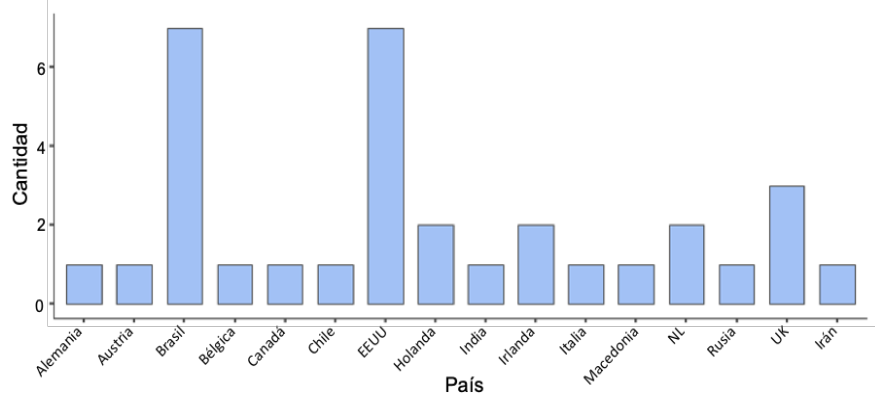

Figura 3. Países de origen de las publicaciones.

Respecto a los participantes con fisura del paladar, el promedio de participantes entre todos los estudios fue de 87 personas, con un rango que varía de 10 personas con fisura del paladar en un estudio, hasta 647 personas, en el estudio con la mayor muestra observada. El rango de edad de los sujetos fue de 2.5 a 83 años. En cuanto a la edad de la cirugía primaria solo $9(27.3 \%)$ estudios reportaron este dato, los cuales señalan un rango de 3 meses a 4 años. Por su parte, la presencia de fístulas fue reportado en solo 2 (6\%) estudios. Por último, respecto al grupo control, 6 de las investigaciones incluyeron participantes sin fisura, de los cuales el promedio de personas fue de 7 sujetos, con un rango de edad que varió desde 2 a los 35 años. 
Tabla 3. Resumen de la evaluación perceptual de la función velofaríngea durante el habla.

\begin{tabular}{|c|c|c|c|c|c|c|c|c|}
\hline Autores (año) & Objetivo del estudio & $\begin{array}{l}\text { Parámetros } \\
\text { perceptuales } \\
\text { evaluados }\end{array}$ & Escala: IAI; EMD; EVA & $\begin{array}{l}\text { Muestra de habla: } \\
\text { espontánea; } \\
\text { automática; } \\
\text { oraciones; palabras; } \\
\text { sílabas; sonidos }\end{array}$ & $\begin{array}{l}\text { Número y tipo de } \\
\text { evaluador: flgo. } \\
\text { experto; flgo. } \\
\text { inexperto; oyente no } \\
\text { entrenado }\end{array}$ & $\begin{array}{l}\text { Análisis: } \\
\text { en vivo; } \\
\text { audio; } \\
\text { video }\end{array}$ & $\begin{array}{l}\text { Evaluación } \\
\text { instrumental: } \\
\text { VNF; VFC; } \\
\text { nasometría }\end{array}$ & Principales resultados \\
\hline $\begin{array}{l}\text { Nellis et al., } \\
\text { (1992) }\end{array}$ & $\begin{array}{l}\text { Relacionar los valores de } \\
\text { nasalancia y el juicio } \\
\text { perceptual de nasalidad en } \\
\text { individuos con colgajo } \\
\text { faríngeo. }\end{array}$ & $\begin{array}{l}\text { - Hipernasalidad } \\
\text { - Hiponasalidad }\end{array}$ & $\begin{array}{l}\text { - IAI: } 1-6 \text { pts (ausente, leve, } \\
\text { leve-moderada, moderada, } \\
\text { moderada-severa o severa) }\end{array}$ & - Oraciones & - 10 flgo. experto & - Audio & - Nasometría & $\begin{array}{l}\text { Las correlaciones entre el juicio perceptual } \\
\text { de hipernasalidad y la nasalancia no fueron } \\
\text { significativas. }\end{array}$ \\
\hline $\begin{array}{l}\text { Williams et al. } \\
\text { (1998) }\end{array}$ & $\begin{array}{l}\text { Evaluar los resultados de la } \\
\text { palatoplastia primaria } \\
\text { realizada por el método de } \\
\text { Larisa Y. Frolova, M.D. } \\
\text { (1971). }\end{array}$ & $\begin{array}{l}\text { - Hipernasalidad } \\
\text { - Hiponasalidad } \\
\text { - Emisión nasal }\end{array}$ & - IAI: presente o ausente & - Palabras & -2 flgo. experto & - En vivo & NR & $\begin{array}{l}\text { En la evaluación perceptual } 55.5 \% \text { de los } \\
\text { participantes fue clasificado con una } \\
\text { resonancia normal. En tanto un } 9.5 \% \text { de los } \\
\text { sujetos presentó hiponasalidad. }\end{array}$ \\
\hline \multirow[t]{2}{*}{$\begin{array}{l}\text { Keuning et al., } \\
\text { (1999) }\end{array}$} & \multirow[t]{2}{*}{$\begin{array}{l}\text { Evaluar la confiabilidad de } \\
\text { las calificaciones de } \\
\text { percepción de cuatro tipos } \\
\text { de muestras de habla. }\end{array}$} & $\begin{array}{l}\text { - Hipernasalidad } \\
\text { - Emisión nasal } \\
\text { - AC }\end{array}$ & $\begin{array}{l}\text { - EVA: 0-100 mm (normal a } \\
\text { extremadamente desviado) }\end{array}$ & \multirow[t]{2}{*}{ - Oraciones } & \multirow[t]{2}{*}{$\begin{array}{l}-3 \text { flgo. experto } \\
-2 \text { flgo. inexperto } \\
-1 \text { oyente no } \\
\text { entrenado (cirujano) }\end{array}$} & \multirow[t]{2}{*}{ - Audio } & \multirow[t]{2}{*}{ NR } & \multirow{2}{*}{$\begin{array}{l}\text { Los jueces de la evaluación perceptual } \\
\text { difieren en gran medida en el rango que } \\
\text { usaban en su calificación. Además la } \\
\text { experiencia de los fonoaudiólogos expertos } \\
\text { no garantizó una alta confiabilidad. }\end{array}$} \\
\hline & & - Hiponasalidad & $\begin{array}{l}\text { - IAI: } 1-3 \text { pts (a veces, } \\
\text { siempre presente o ausente) }\end{array}$ & & & & & \\
\hline \multirow[t]{3}{*}{$\begin{array}{l}\text { Sell et al. } \\
(2001)\end{array}$} & \multirow[t]{3}{*}{$\begin{array}{l}\text { Describir los resultados de } \\
\text { habla en niños con FLAP } \\
\text { unilateral, intervenidos en } \\
\text { Reino Unido. }\end{array}$} & $\begin{array}{l}\text { - Hipernasalidad } \\
\text { - Hiponasalidad } \\
\text { - Emisión nasal } \\
\text { - Turbulencia nasal }\end{array}$ & $\begin{array}{l}\text { - IAI: } 0-4 \text { pts (ausente, leve y } \\
\text { ocasional, leve y consistente, } \\
\text { moderada y consistente o } \\
\text { severa y consistente) }\end{array}$ & \multirow[t]{3}{*}{$\begin{array}{l}\text { - Automática } \\
\text { - Espontánea }\end{array}$} & \multirow[t]{3}{*}{ - 2 flgo. experto } & \multirow[t]{3}{*}{$\begin{array}{l}\text { - Audio } \\
\text { - Video }\end{array}$} & \multirow[t]{3}{*}{ NR } & \multirow{3}{*}{$\begin{array}{l}\text { Los hallazgos de la evaluación perceptual } \\
\text { sugieren que, las cirugías primarias para } \\
\text { niños con FLAP están produciendo malos } \\
\text { resultados de habla y que la terapia } \\
\text { fonoaudiológica no cubre las necesidades de } \\
\text { los niños. }\end{array}$} \\
\hline & & - Movimiento facial & - IAI: ausente o presente & & & & & \\
\hline & & - Inteligibilidad & $\begin{array}{l}\text { - IAI: } 0-5 \text { pts (normal, a } \\
\text { imposible de entender) }\end{array}$ & & & & & \\
\hline $\begin{array}{l}\text { Keuning et al. } \\
\text { (2002) }\end{array}$ & $\begin{array}{l}\text { Correlacionar el valor de } \\
\text { nasalancia y la evaluación } \\
\text { perceptual de varios aspectos } \\
\text { del habla en hablantes con } \\
\text { IVF. }\end{array}$ & $\begin{array}{l}\text { - Grado general de } \\
\text { severidad } \\
\text { - Hipernasalidad } \\
\text { - Emisión nasal } \\
\text { - Inteligibilidad } \\
\text { - AC }\end{array}$ & $\begin{array}{l}\text { - EVA: 0-100 mm (normal a } \\
\text { extremadamente desviado) }\end{array}$ & $\mathrm{NE}$ & - 6 flgo. experto & - Audio & - Nasometría & $\begin{array}{l}\text { Baja correlación entre la nasalancia y la } \\
\text { percepción de hipernasalidad. En tanto, el } \\
\text { grado general de severidad pareció estar } \\
\text { determinado por la inteligibilidad. }\end{array}$ \\
\hline
\end{tabular}




\begin{tabular}{|c|c|c|c|c|c|c|c|c|}
\hline & & - Hiponasalidad & $\begin{array}{l}\text { - IAI: ausente, a veces } \\
\text { presente o siempre presente }\end{array}$ & & & & & $\begin{array}{l}\text { La experiencia mostró una alta correlación } \\
\text { entre la medición instrumental y la }\end{array}$ \\
\hline & & - Calidad vocal & $\begin{array}{l}\text { - IAI: buena, moderada o } \\
\text { mala }\end{array}$ & & & & & \\
\hline \multirow[t]{2}{*}{$\begin{array}{l}\text { Konst et al. } \\
\text { (2003) }\end{array}$} & \multirow[t]{2}{*}{$\begin{array}{l}\text { Investigar el efecto de la } \\
\text { ortopedia infantil utilizada } \\
\text { en el primer año de vida, } \\
\text { sobre las características del } \\
\text { habla en niños con FLAP, } \\
\text { mediante una evaluación } \\
\text { perceptual. }\end{array}$} & $\begin{array}{l}\text { - Lugar de } \\
\text { articulación } \\
\text { - Voz hiperkinética } \\
\text { - Hipernasalidad } \\
\text { - Emisión nasal } \\
\text { - Fricativa nasal } \\
\text { - Resoplido nasal } \\
\text { - Realización nasal } \\
\text { - Corrección de la } \\
\text { articulación } \\
\text { - Inteligibilidad }\end{array}$ & - IAI: $1-7 \mathrm{pts}$ & \multirow[t]{2}{*}{ - Espontánea } & \multirow[t]{2}{*}{ - 5 flgo. experto } & \multirow[t]{2}{*}{ - Audio } & \multirow[t]{2}{*}{ NR } & \multirow[t]{2}{*}{$\begin{array}{l}\text { La confiabilidad y consistencia de las escalas } \\
\text { de evaluación perceptual fue buena. La } \\
\text { escala de calificación de inteligibilidad fue la } \\
\text { única característica del habla que distinguió } \\
\text { a los niños con ortopedia infantil. }\end{array}$} \\
\hline & & - Impresión general & - IAI: $1-10 \mathrm{pts}$ & & & & & \\
\hline $\begin{array}{l}\text { Lewis et al. } \\
\text { (2003) }\end{array}$ & $\begin{array}{l}\text { Evaluar el nivel de } \\
\text { concordancia de la } \\
\text { evaluación perceptual de } \\
\text { nasalidad, y su relación con } \\
\text { los valores de nasalancia, la } \\
\text { experiencia clínica del } \\
\text { oyente y la formación } \\
\text { académica. }\end{array}$ & - Hipernasalidad & $\begin{array}{l}\text { - IAI: } 1-5 \text { pts (resonancia } \\
\text { normal a hipernasalidad } \\
\text { severa) }\end{array}$ & - Oraciones & $\begin{array}{l}-3 \text { flgo. experto } \\
\text { (docente y clínico) } \\
-3 \text { flgo. inexperto } \\
\text { (docente) } \\
-3 \text { flgo. inexperto } \\
\text { (alumno) } \\
-3 \text { oyente no } \\
\text { entrenado (cirujano) }\end{array}$ & - Audio & - Nasometría & $\begin{array}{l}\text { Los niveles de concordancia para la } \\
\text { evaluación perceptual fueron más altos para } \\
\text { los fonoaudiólogos seguidos por los } \\
\text { cirujanos. Los fonoaudiólogos expertos y } \\
\text { cirujanos calificaron la hipernasalidad como } \\
\text { más baja. } \\
\text { Los coeficientes de correlación entre la } \\
\text { nasalancia y la evaluación perceptual fueron } \\
\text { de bajo a moderados. }\end{array}$ \\
\hline $\begin{array}{l}\text { Álvarez et al. } \\
\text { (2004) }\end{array}$ & $\begin{array}{l}\text { Evaluar la validez y } \\
\text { confiabilidad de un }\end{array}$ & - Emisión nasal & $\begin{array}{l}\text { - IAI: } 0-1 \text { pts (ausente o } \\
\text { inconsistente) }\end{array}$ & $\begin{array}{l}\text { - Sílabas } \\
\text { - Palabras }\end{array}$ & - 3 flgo. experto & $\begin{array}{l}\text { - Audio } \\
\text { - Video }\end{array}$ & - VNF & $\begin{array}{l}\text { El protocolo de evaluación se correlacionó } \\
\text { altamente con el hiato obtenido en el examen }\end{array}$ \\
\hline
\end{tabular}




\begin{tabular}{|c|c|c|c|c|c|c|c|c|}
\hline & \multirow[t]{4}{*}{$\begin{array}{l}\text { Protocolo de evaluación para } \\
\text { pacientes con FLAP. }\end{array}$} & $\begin{array}{l}\text { - Emisión nasal } \\
\text { consistente visible } \\
\text { - Emisión nasal } \\
\text { consistente audible } \\
\text { - Turbulencia nasal } \\
\text { - Baja presión } \\
\text { intraoral } \\
\text { - Movimientos } \\
\text { faciales } \\
\text { - Hipernasalidad }\end{array}$ & $\begin{array}{l}\text { - IAI: } 1-3 \text { pts (leve, moderada } \\
\text { o severa) }\end{array}$ & \multirow[t]{4}{*}{$\begin{array}{l}\text { - Oraciones } \\
\text { - Espontánea }\end{array}$} & & & & \multirow[t]{4}{*}{$\begin{array}{l}\text { de VNF. Un puntaje perceptual alto se } \\
\text { relacionó con un alto porcentaje de hiato del } \\
\text { esfínter velofaríngeo. } \\
\text { En el análisis de confiabilidad no se } \\
\text { encontraron diferencias significativas entre } \\
\text { las calificaciones de los oyentes. }\end{array}$} \\
\hline & & $\begin{array}{l}\text { - Resonancia normal/ } \\
\text { Hiponasalidad }\end{array}$ & - IAI: presente o ausente & & & & & \\
\hline & & - Resonancia mixta & $\begin{array}{l}\text { - IAI: } 1-2 \text { pts (presente o } \\
\text { ausente) }\end{array}$ & & & & & \\
\hline & & $-\mathrm{AC}$ & - IAI: presente o ausente & & & & & \\
\hline $\begin{array}{l}\text { Keuning et al. } \\
\text { (2004) }\end{array}$ & $\begin{array}{l}\text { Evaluar el potencial uso } \\
\text { clínico de medidas } \\
\text { compuestas, derivadas de } \\
\text { puntuaciones medias de } \\
\text { nasalancia. }\end{array}$ & $\begin{array}{l}\text { - Grado general de } \\
\text { severidad } \\
\text { - Hipernasalidad } \\
\text { - Emisión nasal } \\
\text { - Inteligibilidad } \\
\text { - AC }\end{array}$ & $\begin{array}{l}\text { - EVA: 0-100 mm (normal a } \\
\text { extremadamente desviado) }\end{array}$ & - Oraciones (párrafo) & - 6 flgo. experto & - Audio & - Nasometría & $\begin{array}{l}\text { La normalización de las puntuaciones de } \\
\text { nasalancia (medidas compuestas) no mejoró } \\
\text { la correlación con los índices de percepción. }\end{array}$ \\
\hline $\begin{array}{l}\text { Paal et al. } \\
\text { (2005) }\end{array}$ & $\begin{array}{l}\text { Evaluar la confiabilidad de } \\
\text { la VNF y la percepción } \\
\text { auditiva, empleados en la } \\
\text { evaluación del habla de } \\
\text { niños con FLAP. }\end{array}$ & $\begin{array}{l}\text { - Nasalidad } \\
\text { - Sitio de } \\
\text { articulación } \\
\text { - Tensión } \\
\text { articulatoria } \\
\text { - Sigmatismo } \\
\text { interdental }\end{array}$ & $\begin{array}{l}\text { - IAI: 0-3 pts (variación } \\
\text { auditiva no perceptible a } \\
\text { variación auditiva distinta) }\end{array}$ & - Palabras & $\begin{array}{l}\text { - } 2 \text { flgo. experto } \\
-2 \text { flgo. inexperto }\end{array}$ & - Video & - VNF & $\begin{array}{l}\text { Los hallazgos visuales y perceptivos de los } \\
\text { evaluadores experimentados fueron } \\
\text { altamente confiables. En tanto, la relación } \\
\text { entre la VNF y la percepción de } \\
\text { hipernasalidad resultó en poca correlación. }\end{array}$ \\
\hline \multirow[t]{2}{*}{$\begin{array}{l}\text { John et al. } \\
\text { (2006) }\end{array}$} & \multirow{2}{*}{$\begin{array}{l}\text { Desarrollar una herramienta } \\
\text { llamada CAPS-A, para la } \\
\text { evaluación del habla en } \\
\text { personas con FLAP, } \\
\text { utilizada para estudios de } \\
\text { auditoria entre centros. }\end{array}$} & - Hipernasalidad & $\begin{array}{l}\text { - IAI: } 0-4 \text { pts (ausente, } \\
\text { mínima, leve, moderada o } \\
\text { severa) }\end{array}$ & \multirow[t]{2}{*}{$\begin{array}{l}\text { - Automática } \\
\text { - Oraciones } \\
\text { - Espontánea }\end{array}$} & \multirow[t]{2}{*}{ - 7 flgo. experto } & \multirow[t]{2}{*}{ - Video } & \multirow[t]{2}{*}{$\begin{array}{l}\text { - Nasometría } \\
\text { - VNF } \\
\text { - VFC }\end{array}$} & \multirow[t]{2}{*}{$\begin{array}{l}\text { CAPS-A es una herramienta aceptable, } \\
\text { válida y confiable para la evaluación } \\
\text { perceptiva del habla en personas con FLAP. }\end{array}$} \\
\hline & & - Hiponasalidad & $\begin{array}{l}\text { - IAI: } 0 \text { - } 2 \text { pts (ausente, leve o } \\
\text { marcada) }\end{array}$ & & & & & \\
\hline
\end{tabular}




\begin{tabular}{|c|c|c|c|c|c|c|c|c|}
\hline & & - Movimiento facial & - IAI: ausente o presente & & & & & \\
\hline \multirow[t]{4}{*}{$\begin{array}{l}\text { Sweeney \& } \\
\text { Sell (2008) }\end{array}$} & $\begin{array}{l}\text { Evaluar la relación entre la } \\
\text { evaluación perceptual } \\
\text { (Temple Street Scale) y la }\end{array}$ & - Hipernasalidad & $\begin{array}{l}\text { - IAI: } 0-5 \text { pts (ausente a } \\
\text { severa); consistente o } \\
\text { inconsistente }\end{array}$ & \multirow{4}{*}{$\begin{array}{l}\text { - Palabras } \\
\text { - Sílabas } \\
\text { - Oraciones } \\
\text { - Espontánea } \\
\text { - Automática }\end{array}$} & \multirow[t]{4}{*}{$\begin{array}{l}\text { - } 1 \text { flgo. experto } \\
-2 \text { flgo. inexperto }\end{array}$} & \multirow[t]{4}{*}{ - En vivo } & \multirow[t]{4}{*}{ - Nasometría } & \multirow{4}{*}{$\begin{array}{l}\text { La escala Temple Street y el nasómetro son } \\
\text { herramientas clínicas válidas para la } \\
\text { evaluación de la nasalidad cuando se utiliza } \\
\text { una muestra de habla cuidadosamente } \\
\text { construida. Se destaca la necesidad de } \\
\text { utilizar la nasometría como complemento de } \\
\text { la evaluación perceptiva. }\end{array}$} \\
\hline & $\begin{array}{l}\text { utilizando muestras } \\
\text { controladas. }\end{array}$ & - Hiponasalidad & $\begin{array}{l}\text { - IAI: } 0-3 \text { pts (ausente a } \\
\text { severa); consistente o } \\
\text { inconsistente }\end{array}$ & & & & & \\
\hline & & $\begin{array}{l}\text { - Cul-de-Sac } \\
\text { - Turbulencia } \\
\text { intranasal }\end{array}$ & - IAI: ausente o presente & & & & & \\
\hline & & $\begin{array}{l}\text { - Emisión nasal } \\
\text { - Fricativa nasal } \\
\text { - Turbulencia nasal } \\
\text { - Fricativa } \\
\text { velofaríngea }\end{array}$ & $\begin{array}{l}\text { - IAI: débil o fuerte, frecuente } \\
\text { o infrecuente, consistente o } \\
\text { inconsistente, o fonema } \\
\text { específico }\end{array}$ & & & & & \\
\hline \multirow[t]{2}{*}{$\begin{array}{l}\text { Rullo et al. } \\
\text { (2009) }\end{array}$} & $\begin{array}{l}\text { Examinar los resultados de } \\
\text { habla en un grupo de niños } \\
\text { con FLAP operados por un } \\
\text { mismo cirujano. }\end{array}$ & $\begin{array}{l}\text { - Inteligibilidad } \\
\text { - Hipernasalidad } \\
\text { - Hiponasalidad } \\
\text { - Emisión nasal } \\
\text { - Fricativa faríngea } \\
\text { - Golpe glótico }\end{array}$ & $\begin{array}{l}\text { - IAI: } 0-3 \text { pts (severa y } \\
\text { consistente, moderado, leve y } \\
\text { ocasional o ausente) }\end{array}$ & \multirow[t]{2}{*}{ - Espontánea } & \multirow[t]{2}{*}{ - 1 flgo. experto } & \multirow[t]{2}{*}{ - En vivo } & \multirow[t]{2}{*}{ NR } & \multirow{2}{*}{$\begin{array}{l}\text { La evaluación perceptual mostró que el } \\
\text { desarrollo fonético y fonológico en el niño } \\
\text { con FLAP no solo se debe a las estrategias } \\
\text { quirúrgicas y la experiencia del cirujano, } \\
\text { sino que también está influenciado por la } \\
\text { colaboración del paciente y especialmente de } \\
\text { los padres, la puntualidad de la intervención } \\
\text { fonoaudiológica y por las capacidades del } \\
\text { niño. }\end{array}$} \\
\hline & & - Movimiento facial & $\begin{array}{l}\text { - IAI: 0-3 pts (movimiento } \\
\text { facial, movimento nasal, } \\
\text { dilatación nasal o ausente) }\end{array}$ & & & & & \\
\hline
\end{tabular}




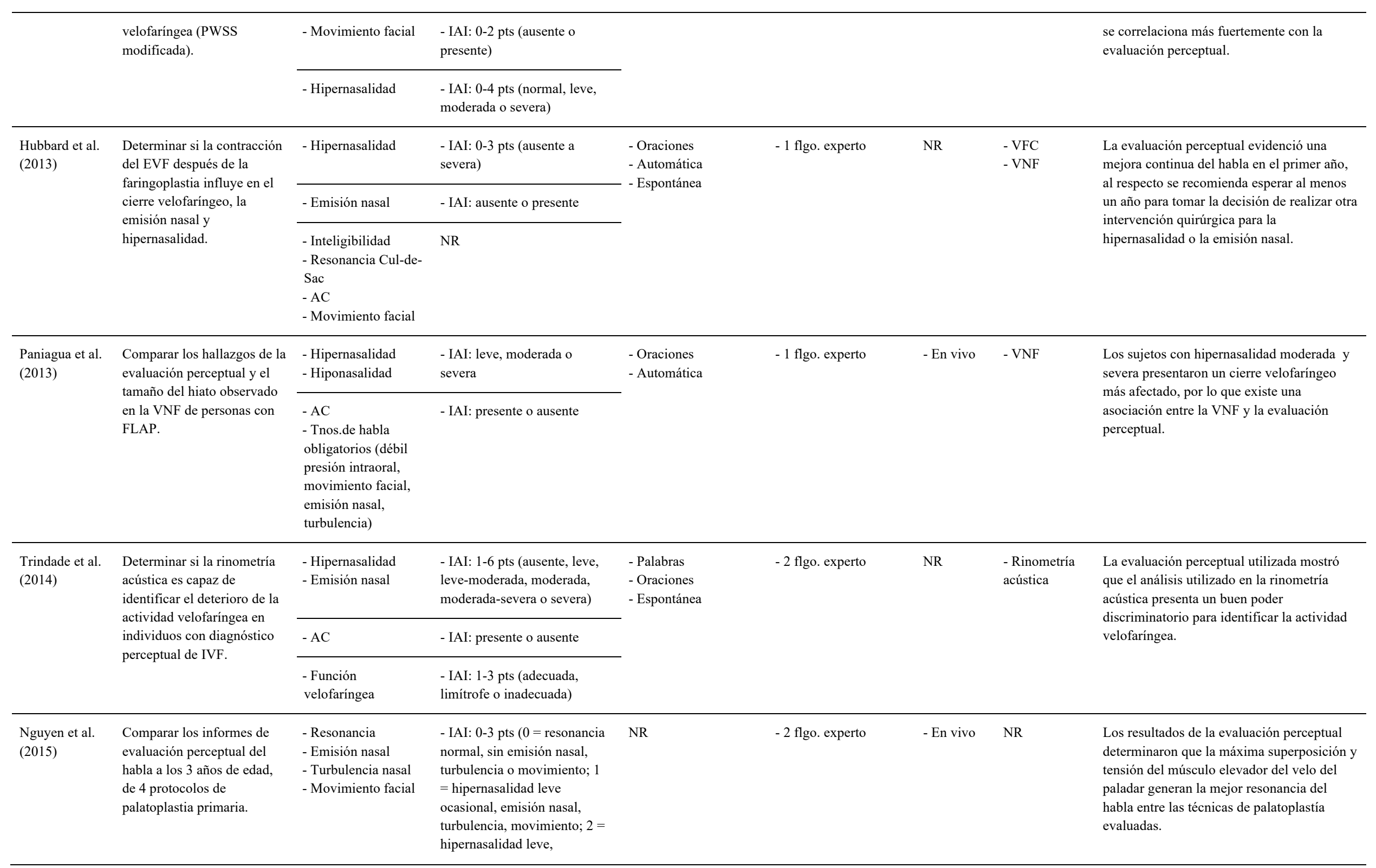


turbulencia nasal intermitente

movimiento: 3 =

hipernasalidad grave asociada

a anomalías anatómicas)

\begin{tabular}{|c|c|c|c|c|c|c|c|c|}
\hline \multirow[t]{2}{*}{$\begin{array}{l}\text { Padilha et al. } \\
\text { (2015) }\end{array}$} & \multirow{2}{*}{$\begin{array}{l}\text { Describir y comparar los } \\
\text { resultados de la evaluación } \\
\text { perceptual de nasalidad } \\
\text { realizada en vivo y por el } \\
\text { análisis de grabaciones. }\end{array}$} & \multirow[t]{2}{*}{ - Hipernasalidad } & \multirow[t]{2}{*}{$\begin{array}{l}\text { - IAI: 0-3 pts (ausente, leve, } \\
\text { moderada o severa) }\end{array}$} & \multicolumn{2}{|l|}{$\begin{array}{l}\text { - Palabras } \\
\text { - Oraciones } \\
\text { - Espontánea }\end{array}$} & - En vivo & NR & \multirow{2}{*}{$\begin{array}{l}\text { El juicio perceptual en vivo puede detectar } \\
\text { mejor la ausencia de hipernasalidad del } \\
\text { habla, así como la hipernasalidad leve, en } \\
\text { comparación con el juicio realizado por } \\
\text { múltiples jueces, a partir de muestras } \\
\text { registradas. }\end{array}$} \\
\hline & & & & - Oraciones & - 3 flgo. experto & - Audio & NR & \\
\hline \multirow[t]{2}{*}{$\begin{array}{l}\text { Scarmagnani } \\
\text { et al. (2015) }\end{array}$} & \multirow{2}{*}{$\begin{array}{l}\text { Correlacionar las } \\
\text { dimensiones del cierre } \\
\text { velofaríngeo con la } \\
\text { evaluación perceptual en } \\
\text { individuos con FLAP } \\
\text { operada. }\end{array}$} & - Hipernasalidad & $\begin{array}{l}\text { - IAI: } 1-4 \text { pts } \\
\text { (ausente/resonancia } \\
\text { equilibrada, leve, moderada o } \\
\text { severa) }\end{array}$ & \multirow[t]{2}{*}{ - Oraciones } & \multirow[t]{2}{*}{ - 3 flgo. experto } & \multirow[t]{2}{*}{ - Audio } & \multirow[t]{2}{*}{$\begin{array}{l}\text { - Evaluación } \\
\text { aerodinámica } \\
\text { (técnica flujo- } \\
\text { presión) }\end{array}$} & \multirow{2}{*}{$\begin{array}{l}\text { El análisis de regresión mostró que las } \\
\text { características perceptivas del habla } \\
\text { contribuyeron significativamente a predecir } \\
\text { el cierre velofaríngeo. } \\
\text { La hipernasalidad y la emisión nasal de aire } \\
\text { se correlacionaron significativamente con el } \\
\text { área velofaríngea. En tanto, la turbulencia } \\
\text { nasal se correlacionó negativamente con el } \\
\text { área velofaríngea. }\end{array}$} \\
\hline & & $\begin{array}{l}\text { - Emisión nasal } \\
\text { - Turbulencia nasal }\end{array}$ & - IAI: presente o ausente & & & & & \\
\hline \multirow[t]{3}{*}{$\begin{array}{l}\text { Sell et al. } \\
\text { (2015) }\end{array}$} & \multirow{3}{*}{$\begin{array}{l}\text { Describir los resultados } \\
\text { perceptuales del estudio } \\
\text { Cleft Care UK (CCUK) y } \\
\text { compararlos con la auditoría } \\
\text { del Clinical Standards } \\
\text { Advisory Group (CSAG) de } \\
1998 .\end{array}$} & $\begin{array}{l}\text { - Inteligibilidad } \\
\text { - Hipernasalidad }\end{array}$ & $\begin{array}{l}\text { - IAI: } 0-4 \text { pts (ausente, } \\
\text { mínima, leve, moderada o } \\
\text { severa) }\end{array}$ & \multirow[t]{3}{*}{ NR } & \multirow[t]{3}{*}{ - 2 flgo. experto } & \multirow[t]{3}{*}{$\begin{array}{l}\text { - Audio } \\
\text { - Video }\end{array}$} & \multirow[t]{3}{*}{ NR } & \multirow{3}{*}{$\begin{array}{l}\text { Los hallazgos perceptuales obtenidos en el } \\
\text { estudio CCUK mostraron una fuerte } \\
\text { evidencia de que los resultados de habla } \\
\text { fueron mejores que los observados en el } \\
\text { CSAG. }\end{array}$} \\
\hline & & $\begin{array}{l}\text { - Hiponasalidad } \\
\text { - Emisión nasal } \\
\text { - Turbulencia nasal }\end{array}$ & $\begin{array}{l}\text { - IAI: } 0-2 \text { pts (ausente, leve o } \\
\text { marcada) }\end{array}$ & & & & & \\
\hline & & $-\mathrm{AC}$ & $\begin{array}{l}\text { - IAI: } 0-2 \text { pts (AC ausente, } 1 \\
\text { o } 2 \text { AC, } 3 \text { o más AC) }\end{array}$ & & & & & \\
\hline $\begin{array}{l}\text { Bettens et al } \\
\text { (2016) }\end{array}$ & $\begin{array}{l}\text { Correlacionar las } \\
\text { puntuaciones del nasality } \\
\text { severity index (NSI) } 2.0 \text { y la } \\
\text { evaluación perceptual de } \\
\text { hipernasalidad. }\end{array}$ & $\begin{array}{l}\text { - Hipernasalidad } \\
\text { - Emisión nasal } \\
\text { - Inteligibilidad }\end{array}$ & $\begin{array}{l}\text { - EVA: } 0-100 \mathrm{~mm} \\
\text { (ausente/normal a } \\
\text { severamente } \\
\text { distorsionado/observado con } \\
\text { frecuencia) }\end{array}$ & $\begin{array}{l}\text { - Espontánea } \\
\text { - Oraciones }\end{array}$ & - 4 flgo. experto & $\begin{array}{l}\text { - Audio } \\
\text { - Video }\end{array}$ & NR & $\begin{array}{l}\text { Se encontró una confiabilidad entre oyentes } \\
\text { e intra oyentes de buena a excelente para la } \\
\text { calificación perceptiva. En tanto, el NSI } 2.0 \\
\text { se correlacionó significativamente con la } \\
\text { hipernasalidad percibida. }\end{array}$ \\
\hline \multirow[t]{2}{*}{$\begin{array}{l}\text { Chapman et } \\
\text { al. (2016) }\end{array}$} & \multirow{2}{*}{$\begin{array}{l}\text { Describir el resultado de } 2 \\
\text { estudios de fiabilidad y } \\
\text { calcular el efecto de } \\
\text { entrenamiento en las }\end{array}$} & $\begin{array}{l}\text { - Inteligibilidad/ } \\
\text { Aceptabilidad }\end{array}$ & - IAI: $0-4$ pts & - Espontánea & - 9 flgo. experto & - Audio & NR & \multirow{2}{*}{$\begin{array}{l}\text { Los hallazgos de este estudio sugirieron que } \\
\text { se podrían obtener mejoras en la } \\
\text { confiabilidad entre evaluadores siguiendo un } \\
\text { programa de entrenamiento sistemático. Sin }\end{array}$} \\
\hline & & - Hipernasalidad & - IAI: $0-4$ pts & - Automática & & - Audio & & \\
\hline
\end{tabular}




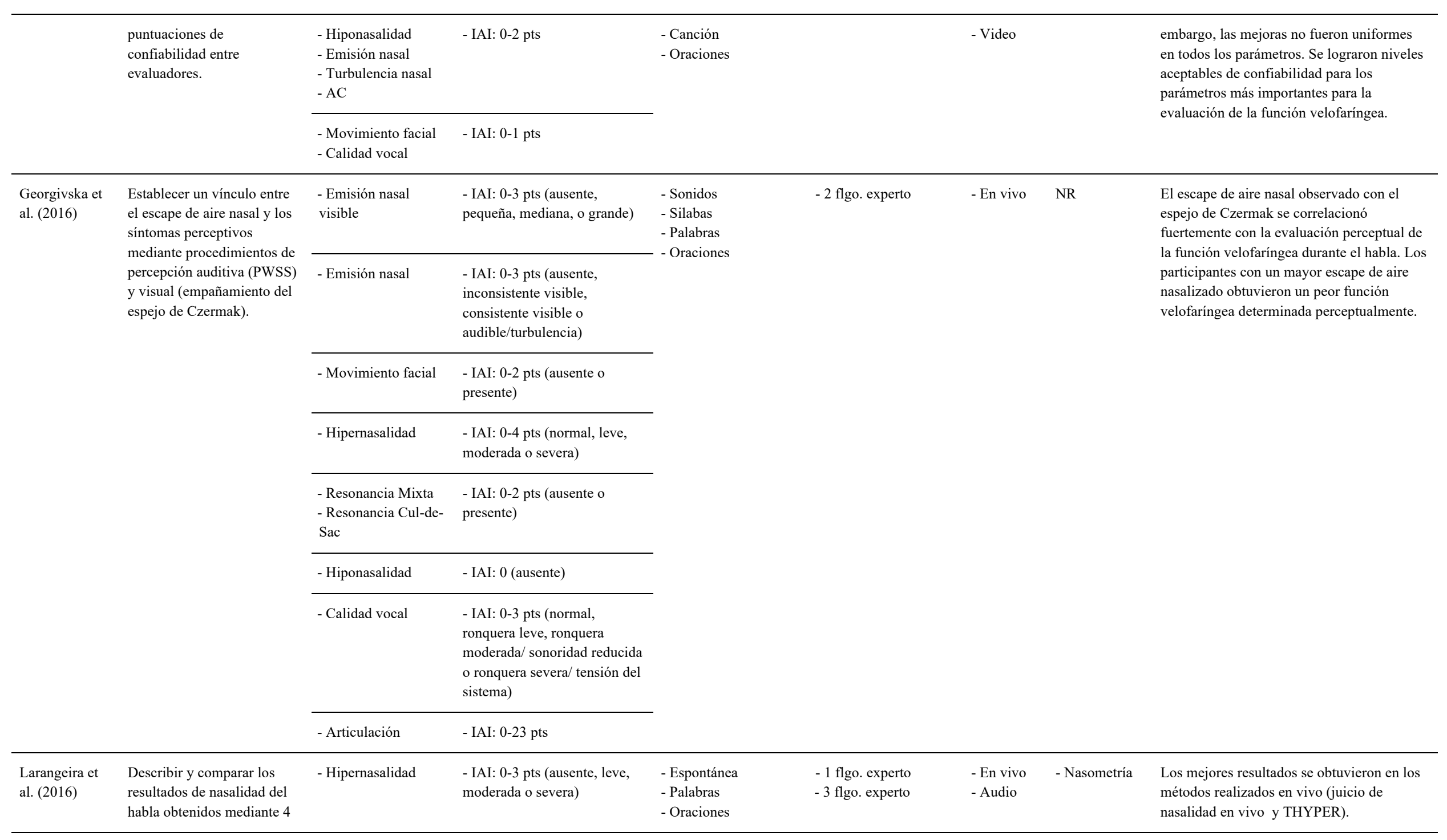




\begin{tabular}{|c|c|c|c|c|c|c|c|c|}
\hline & $\begin{array}{l}\text { modalidades: juicio } \\
\text { perceptivo en vivo; basado } \\
\text { en grabaciones; test de } \\
\text { hipernasalidad (THYPER); y } \\
\text { nasometría. }\end{array}$ & $\begin{array}{l}\text { - THYPER: } 2 \\
\text { repeticiones, sin y } \\
\text { con narinas } \\
\text { obstruidas }\end{array}$ & - Rango 0-10 pts & - 10 palabras & NR & - En vivo & & \\
\hline $\begin{array}{l}\text { Medeiros et } \\
\text { al. (2016) }\end{array}$ & $\begin{array}{l}\text { Investigar la influencia del } \\
\text { tipo de muestra del habla (en } \\
\text { conversación espontánea o } \\
\text { repetición de frases) en el } \\
\text { juicio perceptivo de } \\
\text { hipernasalidad. }\end{array}$ & - Hipernasalidad & $\begin{array}{l}\text { - IAI: } 1-4 \text { pts } \\
\text { (ausente/resonancia normal, } \\
\text { leve, moderada o severa) }\end{array}$ & $\begin{array}{l}\text { - Espontánea } \\
\text { - Oraciones }\end{array}$ & - 3 flgo. experto & - Audio & NR & $\begin{array}{l}\text { La repetición de oraciones mejoró la } \\
\text { confiabilidad intra e inter evaluadores del } \\
\text { juicio perceptual de hipernasalidad. }\end{array}$ \\
\hline $\begin{array}{l}\text { Oliveira et al. } \\
\text { (2016) }\end{array}$ & $\begin{array}{l}\text { Investigar la influencia del } \\
\text { entrenamiento auditivo en la } \\
\text { concordancia de la } \\
\text { evaluación perceptual de la } \\
\text { hipernasalidad de diferentes } \\
\text { oyentes. }\end{array}$ & - Hipernasalidad & $\begin{array}{l}\text { - IAI: } 1-4 \text { pts } \\
\text { (ausente/resonancia normal, } \\
\text { leve, moderada o severa) }\end{array}$ & $\begin{array}{l}\text { - Automática } \\
\text { - Oraciones }\end{array}$ & - 3 flgo. experto & - Audio & NR & $\begin{array}{l}\text { La concordancia para el grado de } \\
\text { hipernasalidad obtenido entre los } 3 \text { oyentes } \\
\text { después del entrenamiento fue } \\
\text { significativamente mayor que la obtenida } \\
\text { antes del entrenamiento. }\end{array}$ \\
\hline \multirow[t]{3}{*}{$\begin{array}{l}\text { Abdali \& } \\
\text { Yaribakht } \\
(2019)\end{array}$} & \multirow{3}{*}{$\begin{array}{l}\text { Evaluar los resultados de la } \\
\text { cirugía para IVF (aumento } \\
\text { de la pared faríngea } \\
\text { posterior), mediante juicio } \\
\text { perceptual (CAPS-A), VNF } \\
\text { y VFC lateral. }\end{array}$} & - Hipernasalidad & $\begin{array}{l}\text { - IAI: 0-4 pts (normal, límite, } \\
\text { leve, moderada o severa) }\end{array}$ & \multirow[t]{3}{*}{ NR } & \multirow[t]{3}{*}{ - 2 flgo. experto } & \multirow[t]{3}{*}{ - En vivo } & \multirow[t]{3}{*}{$\begin{array}{l}-\mathrm{VFC} \\
\text { - VNF }\end{array}$} & \multirow{3}{*}{$\begin{array}{l}\text { La evaluación perceptual y los exámenes } \\
\text { instrumentales mostraron que el aumento de } \\
\text { la pared faríngea posterior con injerto es } \\
\text { eficaz para mejorar la hipernasalidad en } \\
\text { pacientes con un tamaño moderado del } \\
\text { espacio velofaríngeo. }\end{array}$} \\
\hline & & - Hiponasalidad & $\begin{array}{l}\text { - IAI: } 0-2 \text { pts (normal, leve o } \\
\text { significativa) }\end{array}$ & & & & & \\
\hline & & - Emisión nasal & - IAI: ausente o presente & & & & & \\
\hline \multirow[t]{2}{*}{$\begin{array}{l}\text { Aparna et al. } \\
(2019)\end{array}$} & \multirow{2}{*}{$\begin{array}{l}\text { Investigar la función } \\
\text { velofaríngea y los } \\
\text { parámetros de resonancia en } \\
\text { niños después de una } \\
\text { reparación temprana del } \\
\text { paladar. }\end{array}$} & - Hipernasalidad & $\begin{array}{l}\text { - IAI: 0-3 pts (normal, leve, } \\
\text { moderada o severa) }\end{array}$ & \multirow[t]{2}{*}{$\begin{array}{l}\text { - Sílabas } \\
\text { - Oraciones }\end{array}$} & \multirow[t]{2}{*}{ - 3 flgo. experto } & \multirow[t]{2}{*}{$\begin{array}{l}\text { - Audio } \\
\text { - Video }\end{array}$} & \multirow[t]{2}{*}{ - VFC } & \multirow{2}{*}{$\begin{array}{l}\text { Se encontró una buena correlación entre la } \\
\text { tasa de cierre observada en la VFC y la } \\
\text { hipernasalidad evaluada perceptualmente. El } \\
\text { análisis de las imágenes de VFC indicó que } \\
\text { el } 48 \% \text { de los niños tenía cierre completo y } \\
\text { el } 52 \% \text { tenía una resonancia perceptualmente } \\
\text { normal. }\end{array}$} \\
\hline & & - Emisión nasal & $\begin{array}{l}\text { - IAI: } 0-1 \text { pts (ausente, o } \\
\text { presente de forma frecuente o } \\
\text { inconsistente) }\end{array}$ & & & & & \\
\hline
\end{tabular}




\begin{tabular}{|c|c|c|c|c|c|c|c|c|}
\hline \multirow[t]{2}{*}{$\begin{array}{l}\text { de Boer et al. } \\
(2020)\end{array}$} & \multirow{2}{*}{$\begin{array}{l}\text { Evaluar si la pre } \\
\text { clasificación basada en el } \\
\text { equilibrio de los trastornos } \\
\text { del equilibrio oral-nasal } \\
\text { mejora la concordancia del } \\
\text { oyente. }\end{array}$} & - Hipernasalidad & $\begin{array}{l}\text { - IAI: } 0-3 \text { pts (ausente, leve, } \\
\text { moderada o severa) } \\
\text { - EVA }\end{array}$ & \multirow[t]{2}{*}{ - Oraciones } & \multirow[t]{2}{*}{ - 3 flgo. experto } & \multirow[t]{2}{*}{ - Audio } & \multirow[t]{2}{*}{ - Nasometria } & \multirow{2}{*}{$\begin{array}{l}\text { La preclasificación de los trastornos del } \\
\text { equilibrio oral-nasal basada en puntuaciones } \\
\text { de equilibrio puede ayudar a los oyentes a } \\
\text { lograr una mejor precisión diagnóstica y un } \\
\text { mayor acuerdo. La concordancia entre la } \\
\text { evaluación perceptual y la nasalancia } \\
\text { aumento de } 45,1 \text { a } 67,1 \% \text {, mientras que la } \\
\text { concordancia entre oyentes aumento de } 36,7 \\
\text { a } 85.4 \% \text {. }\end{array}$} \\
\hline & & - Hiponasalidad & $\begin{array}{l}\text { - IAI: } 0-2 \text { pts (ausente, leve, } \\
\text { moderada/severa) } \\
\text { - EVA }\end{array}$ & & & & & \\
\hline
\end{tabular}

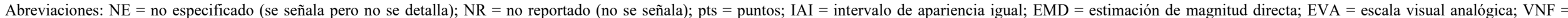

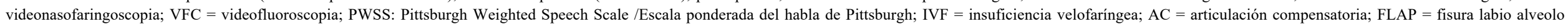
palatina. 
En relación a los parámetros perceptuales evaluados, la figura 4 muestra que el parámetro más evaluado es la hipernasalidad $(\mathrm{N}=31)$ seguido de la emisión nasal $(\mathrm{N}=23)$ y la articulación compensatoria asociada a IVF $(\mathrm{N}=9)$. Mientras que, los parámetros menos evaluados fueron, la resonancia mixta $(\mathrm{N}=2)$, la baja presión intraoral $(\mathrm{N}=2)$, el grado de severidad $(\mathrm{N}=2)$, el sitio de articulación $(\mathrm{N}=2)$, la emisión nasal visible $(\mathrm{N}=2)$ y la fricativa nasal $(\mathrm{N}=2)$.

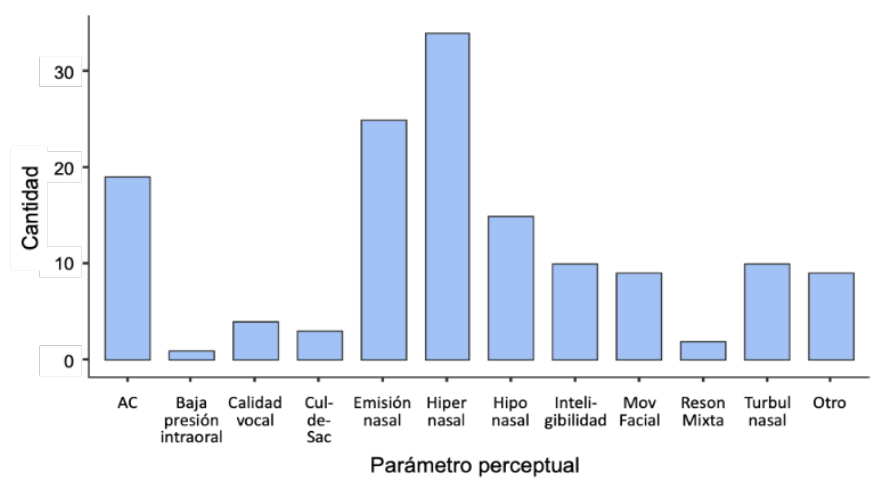

Figura 4. Parámetros perceptuales.

Por su parte, como se observa en la figura 5, la escala de evaluación más utilizada correspondió a la de intervalo de apariencia igual - IAI $(\mathrm{N}=31)$, seguido de la escala visual analógica - EVA $(\mathrm{N}=5)$. En tanto, 1 estudio utilizó la escala de estimación de magnitud directa - EMD.

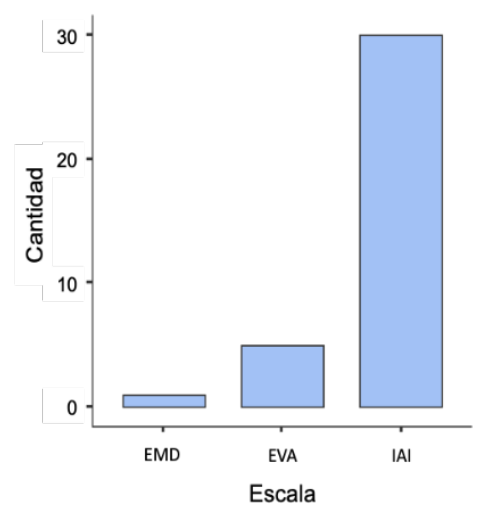

Figura 5. Escala de evaluación.

En la figura 6 se observa que la muestra de habla más utilizada para la evaluación perceptual fueron las oraciones o frases
$(\mathrm{N}=24)$, mientras que otras muestras utilizadas fueron el habla espontánea $(\mathrm{N}=14)$, palabras aisladas $(\mathrm{N}=8)$ y el habla automática $(\mathrm{N}=7)$. En tanto, algunos estudios también incluyeron sonidos aislados, sílabas y en un caso una canción.

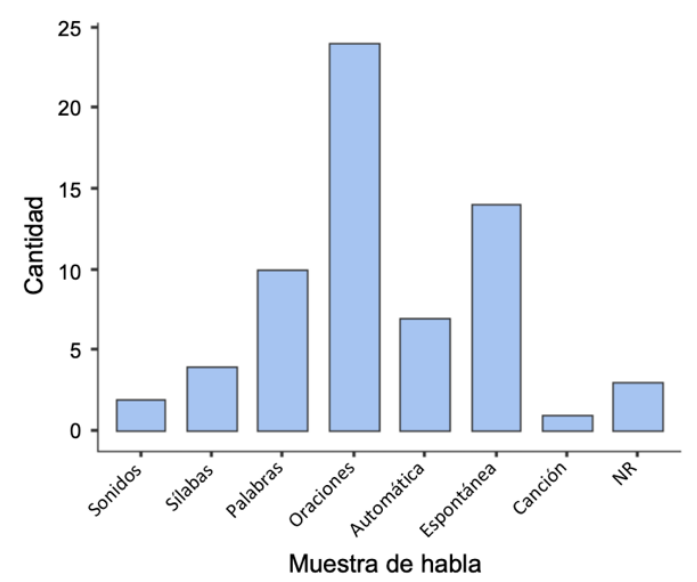

Figura 6. Muestra de habla.

En cuanto al tipo de evaluador, en la mayoría de los estudios se utilizó un fonoaudiólogo o terapeuta del habla experto o entrenado $(\mathrm{N}=33)$. Mientras que, 5 estudios incluyeron fonoaudiólogos inexpertos en la evaluación perceptual de personas con fisura del paladar y 2 estudios a oyentes no fonoaudiólogos, sin ningún tipo de entrenamiento o formación en el área.

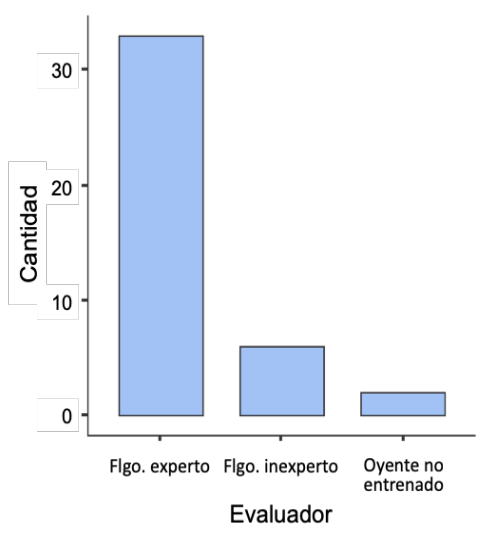

Figura 7. Tipo de evaluador. 
En la figura 8 se muestra el tipo de análisis, al respecto la mayoría de los estudios utilizaron grabaciones de audio para la evaluación perceptual $(\mathrm{N}=20)$. Sin embargo, un número importante realizó las evaluaciones perceptuales en vivo $(\mathrm{N}=9)$.

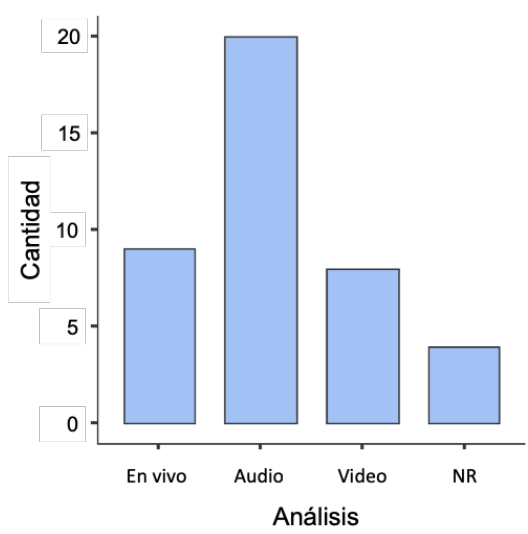

Figura 8. Tipo de análisis.

Por último, la mayoría de los estudios no reportó la realización de una evaluación instrumental. En tanto, los estudios que sí utilizaron una evaluación instrumental para complementar la evaluación perceptual, emplearon principalmente la nasometría $(\mathrm{N}=9)$, la videonasofaringoscopia $(\mathrm{N}=8)$ y la videofluoroscopia $(\mathrm{N}=6)$.

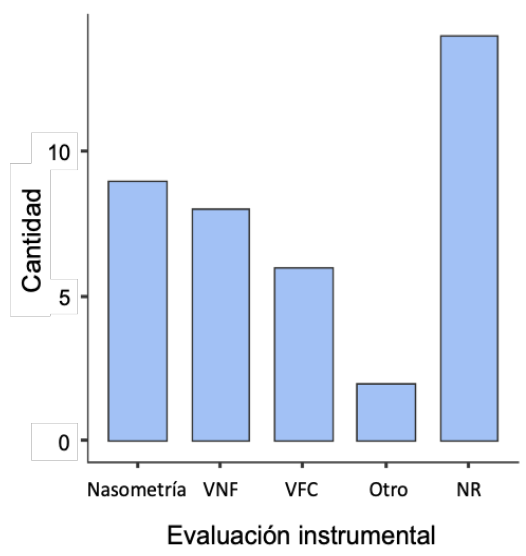

Figura 9. Evaluación instrumental.

\section{DISCUSIÓN}

Se realizó una revisión integradora de literatura con el objetivo de describir los parámetros y procedimientos realizados durante la evaluación perceptual de la insuficiencia velofaríngea en personas con fisura de paladar.

Los parámetros perceptuales más utilizados en los 33 estudios corresponden a: (1) hipernasalidad, la que se define como "cualquier aumento anormal de la resonancia nasal durante la producción del habla que se percibe más fácilmente en las vocales y consonantes sonoras" (John et al., 2006), (2) emisión nasal, definida como "cualquier escape audible anormal o inapropiado de la cavidad nasal que acompaña a la producción de consonantes de presión" (John et al., 2006) y (3) la articulación compensatoria (AC) asociada a IVF, la que corresponde a alteraciones articulatorias "mal adaptadas" originadas inicialmente a consecuencia de la fisura del paladar y que afectan principalmente la producción de sonidos de alta presión: oclusivos y fricativos. Respecto a esta última, es importante señalar que la $\mathrm{AC}$ no es una consecuencia obligatoria de la IVF, como lo son la emisión nasal y la hipernasalidad, sin embargo, la presencia de AC impide la evaluación perceptual de la insuficiencia velofaríngea, debido a que el mecanismo velofaríngeo no está funcionando correctamente. Debido a lo anterior, es importante incluir su evaluación en el juicio perceptual de la IVF, con el objetivo de realizar tratamiento fonoaudiológico para su corrección (Palomares \& Inostroza-Allende, 2019).

Los tres parámetros señalados previamente, son considerados en los parámetros universales para informar resultados de habla en personas con fisura del paladar descritos por Henningsson et al. (2008). Además, Henningsson et al. (2008) consideran la hiponasalidad, los trastornos de voz, la turbulencia nasal, la inteligibilidad del habla y la aceptabilidad del habla, los que también fueron descritos en algunos de los estudios identificados en esta revisión (Abdali \& Yaribakht, 2019; Álvarez et al., 2004; Bettens et al., 2016; Chapman et al., 2016; de Boer et al., 2020; Georgievska-Jancheska et al., 2016; Hubbard et al., 2013; John et al., 2006; Keuning et al., 1999, 2002, 2004; Konst et al., 2003; Kummer et al., 2003; Lipira et al., 2011; Nellis et al., 1992; Nguyen et al., 2015; Paniagua et al., 2013; Rullo et al., 2009; Scarmagnani et al., 2015; Sell et al., 2001, 2015; Sweeney \& Sell, 2008; Williams et al., 1998).

En relación a los procedimientos, en esta revisión las escalas para evaluar cada parámetro fueron clasificadas en: escala de intervalo de apariencia igual (IAI), en la cual los oyentes dividen las sensaciones en categorías discretas; estimación de magnitud 
directa (EMD), en la que los oyentes realizan un juicio mediante la estimación de razones a partir de una referencia; y escala visual analógica (EVA), donde los oyentes califican su percepción en una línea de $100 \mathrm{~mm}$ (Brancamp et al., 2010; Keuning et al., 2004). Al respecto, los hallazgos de esta revisión mostraron un mayor uso de la escala de IAI (alrededor del 90\% de los estudios), la cual también es utilizada por Henningsson et al. (2008) para el reporte de los resultados de habla en personas con fisura del paladar. Al respecto, una revisión previa describió que un $74 \%$ de los estudios aplicó la escala de IAI en la evaluación perceptual del habla en personas con fisura del paladar (Lohmander \& Olsson, 2004), inferior a lo observado en la presente revisión donde un $90 \%$ de las investigaciones utilizó esta escala. En tanto, la EVA fue utilizada en sólo 5 de los 33 estudios analizados en esta revisión, a pesar de haber mostrado validez y confiabilidad para el juicio perceptual en personas con fisura del paladar (Baylis et al., 2015; Whitehill et al., 2007). Por su parte, la EMD demostró ser la escala menos utilizada, lo cual puede ocurrir debido a la dificultad de establecer una referencia que permita evaluar cada parámetro.

En cuanto a la muestra de habla utilizada para el análisis perceptual, las oraciones fueron el parámetro más reportado, según Kummer (2016), este tipo de muestra es considerada una de las más útiles, ya que tiene la ventaja de evaluar de forma rápida y directa la producción y las alteraciones de sonidos específicos. Por otra parte, Henningsson et al. (2008), considera palabras aisladas y oraciones para la evaluación de hipernasalidad, emisión nasal audible y/o turbulencia nasal y los errores en la producción de consonantes, mientras que para la hiponasalidad solo utiliza oraciones, y para los trastornos de la voz, la inteligibilidad y la aceptabilidad del habla emplea muestras de habla espontánea. Al respecto, se debe destacar que es importante que la evaluación perceptual en personas con fisura del paladar incluya palabras, oraciones y muestras de habla espontánea que permitan evaluar sonidos de alta presión $(/ \mathrm{p} /, / \mathrm{t} /, / \mathrm{k} /, / \mathrm{f} /, / \mathrm{s} / \mathrm{y} / / \mathrm{t} /)$, comúnmente afectados en personas con fisura (Palomares-Aguilera et al., 2021).

Las evaluaciones en personas con fisura del paladar fueron realizadas principalmente por fonoaudiólogos o terapeutas de habla expertos o entrenados. En tanto, un número limitado de estudios incluyó fonoaudiólogos y oyentes (no fonoaudiólogos) inexpertos y sin entrenamiento. Los hallazgos de esta revisión muestran la valoración de la experiencia del juez en la evaluación perceptual de la IVF en la mayoría de los estudios, la importancia de dicha experiencia ha sido reportada previamente en juicios perceptuales relacionados a patologías vocales (Farías, 2016). Al respecto, Henningsson et al. (2008) también sugieren que la evaluación debe ser realizada por un fonoaudiólogo experto. Sin embargo, se considera relevante la necesidad de más estudios con oyentes (no fonoaudiólogos) inexpertos y sin entrenamiento, ya que esto puede evidenciar de qué manera influye la insuficiencia velofaríngea frente a personas legas. Ello, aporta información sobre la inteligibilidad y aceptabilidad del mensaje en contextos reales, estableciendo así resultados que reflejen la competencia comunicativa en las personas con IVF secundaria a fisura del paladar (Brunnegård et al., 2009). Por otra parte, es importante considerar algunos factores del examinador que pueden influir en los juicios de percepción, como las diferencias individuales debido a la experiencia, los hábitos de percepción, los prejuicios, entre otros (Kreiman et al., 1993).

Respecto del tipo de análisis (en vivo, audio y video) utilizados para el juicio perceptual, Padilha et al. (2015) describe que el análisis más adecuado es la evaluación en vivo, pues permite observar la competencia del usuario en un contexto comunicativo funcional, siendo posible identificar la presencia de movimientos faciales asociados a las IVF u otros, como la articulación compensatoria y los trastornos de los sonidos del habla (TSH) de tipo articulatorio. Sin embargo, en la presente revisión la mayoría de los estudios utilizó grabaciones de audio. Cabe destacar que las grabaciones de audio tienen la ventaja de ser almacenadas y reproducidas nuevamente, para así por ejemplo evaluar la concordancia entre e intra examinadores (Álvarez et al., 2004; Aparna et al., 2019; Bettens et al., 2016; Chapman et al., 2016; de Boer et al., 2020; Keuning et al., 1999, 2002, 2004; Konst et al., 2003; Larangeira et al., 2016; Lee et al., 2009; Lewis et al., 2003; Medeiros et al., 2016; Nellis et al., 1992; Oliveira et al., 2016; Padilha et al., 2015; Scarmagnani et al., 2015; Sell et al., 2001, 2015; Sinko et al., 2017), y comparar resultados luego de intervenciones quirúrgicas y protésicas para la IVF o de tratamiento fonoaudiológico para la AC (Abdali \& Yaribakht, 2019; Aparna et al., 2019; Konst et al., 2003; Rullo et al., 2009; Sell et al., 2001; Trindade et al., 2014). Por otra parte, algunos estudios incluyeron el análisis mediante video, el cual tiene la ventaja de mostrar los movimientos faciales asociados a IVF y la participación de las estructuras fonoarticulatorias durante el habla, lo que permite identificar con mayor facilidad la AC y los TSH articulatorios (Álvarez et al., 2004; Aparna et al., 2019; Bettens et al., 2016; Chapman et al., 2016; John et al., 2006; Paal et al., 2005; Sell et al., 2015; Sinko et al., 2017). De acuerdo con lo anterior, se sugiere que los equipos multidisciplinarios realicen evaluaciones en vivo y complementarlas con grabaciones de audio y video en buena calidad, ya que esto último también puede interferir en el juicio perceptual (Padilha et al., 2015). 
El juicio perceptual se ha considerado como el procedimiento gold standard para la evaluación de la IVF (Larangeira et al., 2016; Sell, 2005). Sin embargo, es importante destacar la realización de evaluaciones instrumentales como la videofluoroscopia y videonasofaringoscopia para caracterizar el defecto anatómico y realizar la planificación quirúrgica o protésica de cada caso (Abdali \& Yaribakht, 2019; Álvarez et al., 2004; Aparna et al., 2019; Hubbard et al., 2013; John et al., 2006; Kummer et al., 2003; Lipira et al., 2011; Paal et al., 2005; Paniagua et al., 2013). Además, la nasometría que permite cuantificar la nasalidad del habla mediante una medida de nasalancia, que corresponde a la cantidad relativa de energía acústica emitida desde la cavidad nasal durante el habla, la cual ha demostrado correlacionarse altamente con el juicio perceptual (de Boer et al., 2020; Larangeira et al., 2016; Sinko et al., 2017; Sweeney \& Sell, 2008). Sin embargo, en esta revisión un bajo número de estudios complementaron el juicio perceptual con alguna de estas evaluaciones instrumentales, lo cual puede estar relacionado a sus altos costos y al bajo trabajo colaborativo entre fonoaudiólogos, otorrinolaringólogos, radiólogos y cirujanos, lo cual se ha descrito en equipos multidisciplinarios de países en vías de desarrollo (Goldschmied et al., 2021).

Es necesario señalar que la evaluación perceptual de la IVF debe complementarse con una evaluación anatómica y funcional de las estructuras fonoarticulatorias, fundamental para identificar la presencia de fístulas, fisura submucosa no diagnosticada, tonsilas palatinas hiperplásicas, frenillo lingual alterado, anomalías dentomaxilares, entre otras. También se debe realizar una caracterización completa del inventario de sonidos, para identificar los sonidos del habla producidos correctamente y aquellos sustituidos, omitidos, distorsionados y con AC. Para luego, determinar de manera independiente la severidad de la $\mathrm{AC}$ en cada sonido, mediante por ejemplo la aplicación de la escala de Pamplona et al. (2005). Además, de complementar lo anterior con el reporte de los usuarios, sus padres/cuidadores $\mathrm{o}$ acompañantes, a través de cuestionarios como el VELO-Spanish, la escala de inteligibilidad en contexto, entre otros (McLeod et al., 2012; Palomares-Aguilera et al., 2021; Skirko et al., 2018). Estos últimos entregan información importante sobre el impacto de la IVF en la calidad de vida de las personas con fisura del paladar y su desempeño comunicativo en contextos funcionales, lo cual es fundamental para la toma de decisiones respecto a tratamientos quirúrgicos y fonoaudiológicos.

Por otra parte, desde una mirada de la Clasificación Internacional del Funcionamiento, de la Discapacidad y de la Salud, (CIF), según los resultados observados en esta revisión, la estructura y función es abordada por el equipo multidisciplinario durante la evaluación perceptual de las alteraciones de habla y función velofaríngea en personas con fisura del paladar. Sin embargo, la evaluación carece de parámetros que puedan abordar aspectos de la actividad y participación, así como conocer las barreras y facilitadores del contexto social del usuario. Lo cual genera un conflicto, ya que, por un lado, existen los aspectos facilitadores que pueden ser impulsados y regulados por el equipo de salud, pero por otro lado existen las barreras que dependen de cada usuario y el contexto social en el que se sitúa y que son fundamentales para el desarrollo de las personas con fisura del paladar. Como menciona Neumann \& Romonath (2012) los prejuicios que se generan en torno al usuario con IVF, que en su mayoría son niños y adolescentes, pueden sobrellevarse a través de redes de apoyo, a las que tengan acceso los padres/cuidadores y usuarios, como fundaciones, organizaciones de usuarios o reuniones de autoayuda. Al respecto, se debe resaltar que la IVF no necesariamente significa un compromiso en la inteligibilidad, sino que más bien en la aceptabilidad del habla, donde los prejuicios del oyente podrían influir en la caracterización de un habla normal a severa, como lo propuesto por Henningsson et al., (2008).

En Chile, el tratamiento multidisciplinario de las personas con fisura labio alveolo palatina está descrito en la Guía GES de Fisura Labiopalatina (MINSAL, 2015). En esta, se recomienda realizar un tratamiento fonoaudiológico para: prevenir, evaluar y tratar alteraciones de habla, lenguaje y voz, considerando las deficiencias anatómicas y funcionales que presenten los usuarios; lograr que la persona tenga un habla, lenguaje y función velofaríngea normal; prevenir y tratar malos hábitos orales y funciones alteradas del sistema estomatognático. Respecto a la evaluación de la IVF recomienda utilizar el protocolo de evaluación de la IVF de Fundación Gantz (Álvarez et al., 2004), el cual incorpora los parámetros más observados en esta revisión, no obstante, varios de los procedimientos descritos en este documento pueden complementar la evaluación perceptual realizada en la actualidad.

Una limitación de esta investigación es que no se realizó una evaluación de los posibles sesgos en los estudios, a pesar de que se utilizó como guía la lista de verificación de la declaración PRISMA para revisiones sistemáticas (Urrútia \& Bonfill, 2010). Por otra parte, los artículos seleccionados no fueron clasificados según su nivel de evidencia como ha sido sugerido previamente para las revisiones integradoras (Souza et al., 2010). Además, la información obtenida respecto a los parámetros debió incluir la descripción de los descriptores para cada uno, como es descrito para la hipernasalidad en los parámetros universales propuestos por Henningsson et al. (2008). En relación con el análisis de los 
datos de esta revisión, una de las limitaciones es que no se evaluó directamente los aspectos de concordancia entre e intra examinadores, y su relación con aspectos de experiencia, entrenamiento y otros, así como la confiabilidad y validez de los juicios perceptuales.

\section{CONCLUSIÓN}

La presente revisión integradora de literatura destaca se centra en la evaluación perceptual de la insuficiencia velofaríngea en personas con fisura del paladar. Se concluye que los parámetros perceptuales más utilizados corresponden a la hipernasalidad, emisión nasal y la articulación compensatoria asociada a IVF. En tanto, los procedimientos más utilizados incluyen principalmente el uso de oraciones como muestra de habla, además de habla espontánea y palabras, evaluadas por un fonoaudiólogo o terapeuta del habla experto o entrenado, principalmente mediante grabaciones de audio y en segundo lugar en vivo, con una baja realización de evaluaciones instrumentales que concuerden con lo perceptual.

\section{REFERENCIAS}

Abdali, H., \& Yaribakht, M. (2019). Assessment of outcomes and complications of posterior pharyngeal wall augmentation with dermal fat graft in patients with Velopharyngeal Insufficiency (VPI) after primary cleft palate repair: A pilot study. JPRAS Open, 19, 6-18. https://doi.org/10.1016/j.jpra.2018.10.003

Agrawal, K. (2009). Cleft palate repair and variations. Indian Journal of Plastic Surgery: Official Publication of the Association of Plastic Surgeons of India, 42, S102-S109. https://doi.org/10.4103/0970-0358.57197

Álvarez, D., Palomares, M., Quezada, V., \& Villena, C. (2004). Evaluación de la insuficiencia velofaríngea: Presentación de un protocolo de evaluación para pacientes portadores de fisura labiopalatina. Revista Chilena de Fonoaudiología, 5(2), 41-55. https://doi.org/10.5354/0719-4692.2004.56601

Aparna, V. S., Pushpavathi, M., \& Bonanthaya, K. (2019). Velopharyngeal Closure and Resonance in Children Following Early Cleft Palate Repair: Outcome Measurement. Indian Journal of Plastic Surgery, 52(02), 201-208. https://doi.org/10.1055/s-0039-1696608

Baylis, A., Chapman, K., Whitehill, T. L., \& The Americleft Speech Group. (2015). Validity and Reliability of Visual Analog Scaling for Assessment of Hypernasality and Audible Nasal Emission in Children With Repaired Cleft Palate. The Cleft Palate-Craniofacial Journal: Official Publication of the American Cleft Palate-Craniofacial Association, 52(6), 660-670. https://doi.org/10.1597/14-040

Becker, M., Svensson, H., Sarnäs, K. V., \& Jacobsson, S. (2000). Von Langenbeck or Wardill procedures for primary palatal repair in patients with isolated cleft palate-Speech results. Scandinavian Journal of Plastic and Reconstructive Surgery and Hand Surgery, 34(1), 27-32. https://doi.org/10.1080/02844310050160141

Bettens, K., De Bodt, M., Maryn, Y., Luyten, A., Wuyts, F. L., \& Van Lierde, K. M. (2016). The relationship between the Nasality Severity Index 2.0 and perceptual judgments of hypernasality. Journal of Communication Disorders, 62, 67-81. https://doi.org/10.1016/j.jcomdis.2016.05.011

Bicknell, S., McFadden, L. R., \& Curran, J. B. (2002). Frequency of pharyngoplasty after primary repair of cleft palate. Journal (Canadian Dental Association), 68(11), 688-692.

Brancamp, T. U., Lewis, K. E., \& Watterson, T. (2010). The Relationship between Nasalance Scores and Nasality Ratings Obtained with Equal Appearing Interval and Direct Magnitude Estimation Scaling Methods. The Cleft Palate-Craniofacial Journal, 47(6), 631-637. https://doi.org/10.1597/09-106

Brunnegård, K., Lohmander, A., \& Doorn, J. van. (2009). Untrained listeners' ratings of speech disorders in a group with cleft palate: A comparison with speech and language pathologists, ratings. International Journal of Language \& Communication Disorders, 44(5), 656-674. https://doi.org/10.1080/13682820802295203

Chapman, K. L., Baylis, A., Trost-Cardamone, J., Cordero, K. N., Dixon, A., Dobbelsteyn, C., Thurmes, A., Wilson, K., Harding-Bell, A., Sweeney, T., Stoddard, G., \& Sell, D. (2016). The Americleft Speech Project: A Training and Reliability Study. The Cleft Palate-Craniofacial Journal, 53(1), 93-108. https://doi.org/10.1597/14-027

Conley, S. F., Gosain, A. K., Marks, S. M., \& Larson, D. L. (1997). Identification and assessment of velopharyngeal inadequacy. American Journal of Otolaryngology, 18(1), 38-46. https://doi.org/10.1016/s0196-0709(97)90047-8

de Boer, G., Marino, V. C. de C., Dutka, J. de C. R., Pegoraro-Krook, M. I., \& Bressmann, T. (2020). Nasalance-Based Preclassification of Oral-Nasal Balance Disorders Results in Higher Agreement of Expert Listeners' Auditory-Perceptual Assessments: Results of a Retrospective Listening Study. The Cleft Palate$\begin{array}{lll}\text { Craniofacial Journal, } & \text { 57(4), }\end{array}$ https://doi.org/10.1177/1055665619873506

Farías, P. (2016). Guía Clínica Para El Especialista En Laringe y Voz - akad. Akadia Internacional. https://www.librosmedicos.cl/producto/guia-clinica-parael-especialista-en-laringe-y-voz-akad/

Georgievska-Jancheska, T., Gjorgova, J., \& Popovska, M. (2016). The Role of the Velopharyngeal Sphincter in the Speech of Patients with Cleft Palate or Cleft Lip and Palate Using Perceptual Methods. Open Access Macedonian Journal of Medical Sciences, 4(4), 674-679. https://doi.org/10.3889/oamjms.2016.137

Goldschmied, K., Palomares, M., Inostroza-Allende, F., Giugliano, C., Alvarez, D., \& Villena, C. (2021). Online Speech Training in Central America and South America. Annual Meeting American Cleft Palate-Craniofacial Association, 58, 1134. https://doi.org/10.1177/1055665621999916

Henningsson, G., Kuehn, D. P., Sell, D., Sweeney, T., Trost-Cardamone, J. E., \& Whitehill, T. L. (2008). Universal Parameters for Reporting Speech Outcomes in Individuals with Cleft Palate. The Cleft Palate-Craniofacial Journal, 45(1), 1-17. https://doi.org/10.1597/06-086.1

Hubbard, B. A., Rice, G., \& Muzaffar, A. R. (2013). Contractility of sphincter pharyngoplasty: Relevance to speech outcomes. Canadian Journal of Plastic Surgery, 21(1), 15-18. https://doi.org/10.1177/229255031302100108 
John, A., Sell, D., Sweeney, T., Harding-Bell, A., \& Williams, A. (2006). The cleft audit protocol for speech-augmented: A validated and reliable measure for auditing cleft speech. The Cleft Palate-Craniofacial Journal: Official Publication of the American Cleft Palate-Craniofacial Association, 43(3), 272-288. https://doi.org/10.1597/04-141.1

Keuning, K. H., Wieneke, G. H., \& Dejonckere, P. H. (1999). The intrajudge reliability of the perceptual rating of cleft palate speech before and after pharyngeal flap surgery: The effect of judges and speech samples. The Cleft Palate-Craniofacial Journal: Official Publication of the American Cleft PalateCraniofacial Association, 36(4), 328-333. https://doi.org/10.1597/1545156919990360328 tirotp 2.3.co 2

Keuning, K. H., Wieneke, G. H., \& Dejonckere, P. H. (2004). Correlation between the perceptual rating of speech in Dutch patients with velopharyngeal insufficiency and composite measures derived from mean nasalance scores. Folia Phoniatrica et Logopaedica, 56(3), 157-164. https://doi.org/10.1159/000076937

Keuning, K. H., Wieneke, G. H., van Wijngaarden, H. A., \& Dejonckere, P. H. (2002). The correlation between nasalance and a differentiated perceptual rating of speech in Dutch patients with velopharyngeal insufficiency. The Cleft PalateCraniofacial Journal: Official Publication of the American Cleft PalateCraniofacial Association, 39(3), 277-284. https://doi.org/10.1597/15451569_2002_039_0277_tcbnaa_2.0.co_2

Konst, E. M., Rietveld, T., Peters, H. F. M., \& Weersink-Braks, H. (2003). Use of a perceptual evaluation instrument to assess the effects of infant orthopedics on the speech of toddlers with cleft lip and palate. The Cleft Palate-Craniofacial Journal: Official Publication of the American Cleft Palate-Craniofacial Association, 40(6), 597-605. https://doi.org/10.1597/1545$15692003 \quad 040 \quad 0597$ uoapei 2.0.co 2

Kreiman, J., Gerratt, B. R., Kempster, G. B., Erman, A., \& Berke, G. S. (1993). Perceptual Evaluation of Voice Quality. Journal of Speech, Language, and Hearing Research, 36(1), 21-40. https://doi.org/10.1044/jshr.3601.21

Kuehn, D. P., \& Moller, K. T. (2000). Speech and Language Issues in the Cleft Palate Population: The State of the Art. The Cleft Palate-Craniofacial Journal, 37(4), 1-35. https://doi.org/10.1597/1545-1569_2000_037_0348_saliit_2.3.co_2

Kummer, A. W. (2011). Disorders of Resonance and Airflow Secondary to Cleft Palate and/or Velopharyngeal Dysfunction. Seminars in Speech and Language, 32(2), 141-149. https://doi.org/10.1055/s-0031-1277716

Kummer, A. W. (2016). Evaluation of Speech and Resonance for Children with Craniofacial Anomalies. Facial Plastic Surgery Clinics of North America, 24(4), 445-451. https://doi.org/10.1016/j.fsc.2016.06.003

Kummer, A. W., Briggs, M., \& Lee, L. (2003). The Relationship between the Characteristics of Speech and Velopharyngeal Gap Size. The Cleft PalateCraniofacial Journal, 40(6), 590-596. https://doi.org/10.1597/15451569_2003_040_0590_trbtco_2.0.co_2

Lam, E., Hundert, S., \& Wilkes, G. H. (2007). Lateral pharyngeal wall and velar movement and tailoring velopharyngeal surgery: Determinants of velopharyngeal incompetence resolution in patients with cleft palate. Plastic and Reconstructive Surgery, 120(2), 495-505. https://doi.org/10.1097/01.prs.0000267438.18295.e4

Larangeira, F. R., Dutka, J. de C. R., Whitaker, M. E., de Souza, O. M. V., Lauris, J. R. P., da Silva, M. J. F., \& Pegoraro-Krook, M. I. (2016). Speech nasality and nasometry in cleft lip and palate. Brazilian Journal of Otorhinolaryngology, 82(3), 326-333. https://doi.org/10.1016/j.bjorl.2015.05.017
Lee, A., Whitehill, T. L., \& Ciocca, V. (2009). Effect of listener training on perceptual judgement of hypernasality. Clinical Linguistics \& Phonetics, 23(5), 319-334. https://doi.org/10.1080/02699200802688596

Lewis, K. E., Watterson, T. L., \& Houghton, S. M. (2003). The influence of listener experience and academic training on ratings of nasality. Journal of Communication Disorders, 36(1), 49-58. https://doi.org/10.1016/S00219924(02)00134-X

Lipira, A. B., Grames, L. M., Molter, D., Govier, D., Kane, A. A., \& Woo, A. S. (2011). Videofluoroscopic and Nasendoscopic Correlates of Speech in Velopharyngeal Dysfunction. The Cleft Palate-Craniofacial Journal, 48(5), 550560. https://doi.org/10.1597/09-203

Lohmander, A., \& Olsson, M. (2004). Methodology for perceptual assessment of speech in patients with cleft palate: A critical review of the literature. The Cleft Palate-Craniofacial Journal: Official Publication of the American Cleft PalateCraniofacial Association, 41(1), 64-70. https://doi.org/10.1597/02-136

Marrinan, E. M., LaBrie, R. A., \& Mulliken, J. B. (1998). Velopharyngeal function in nonsyndromic cleft palate: Relevance of surgical technique, age at repair, and cleft type. The Cleft Palate-Craniofacial Journal: Official Publication of the American Cleft Palate-Craniofacial Association, 35(2), 95-100. https://doi.org/10.1597/1545-1569_1998_035_0095_vfincp_2.3.co_2

Marrinan, E., \& Shprintzer, R. (2006). Cleft palate and craniofacial disorders. En N. Anderson \& G. Shames (Eds.), Human Communication Disorders: An Introduction ( $8^{\mathrm{a}} \quad$ ed., pp. 263-278). Pearson Education. https://www.pearson.com/store/en-ushttps://www.pearson.com/store/p/humancommunication-disorders-an-introduction/P100001318492

McLeod, S., Harrison, L., \& McCormack, J. (2012). Intelligibility in Context Scale Bathurst. Charles Sturt University. https://cdn.csu.edu.au/_data/assets/pdf_file/0010/399970/ICS-English.pdf

Medeiros, M. N. L. de, Fukushiro, A. P., \& Yamashita, R. P. (2016). Influência da amostra de fala na classificação perceptiva da hipernasalidade. CoDAS, 28(3), 289-294. https://doi.org/10.1590/2317-1782/20162015202

Mendes, K. D. S., Silveira, R. C. de C. P., \& Galvão, C. M. (2008). Revisão integrativa: Método de pesquisa para a incorporação de evidências na saúde e na enfermagem. Texto \& Contexto - Enfermagem, 17(4), 758-764. https://doi.org/10.1590/S0104-07072008000400018

Ministerio de Salud [MINSAL]. (2015). Guía clínica AUGE. Fisura Labiopalatina (Serie de las guías clínicas de MINSAL). http://www.bibliotecaminsal.cl/wp/wp-content/uploads/2016/04/guiaFisuraLabioPalatina-2015-CM.pdf

Moon, J. (2004). Anatomy and physiology of normal and disordered velopharyngeal function for speech. En K. R. Bzoch (Ed.), Communicative Disorders Related to Cleft Lip and Palate (pp. 67-98). PRO-ED.

Morris, H., \& Ozanne, A. (2003). Phonetic, phonological, and language skills of children with a cleft palate. The Cleft Palate-Craniofacial Journal: Official Publication of the American Cleft Palate-Craniofacial Association, 40(5), 460 470. https://doi.org/10.1597/1545-1569_2003_040_0460_ppalso_2.0.co_2

Nellis, J. L., Neiman, G. S., \& Lehman, J. A. (1992). Comparison of Nasometer and Listener Judgments of Nasality in the Assessment of Velopharyngeal Function after Pharyngeal Flap Surgery. The Cleft Palate-Craniofacial Journal, 29(2), 157163. https://doi.org/10.1597/1545-1569_1992_029_0157_conalj_2.3.co_2 
Neumann, S., \& Romonath, R. (2012). Application of the International Classification of Functioning, Disability, and Health-Children and Youth Version (ICF-CY) to Cleft Lip and Palate. The Cleft Palate-Craniofacial Journal, 49(3), 325-346. https://doi.org/10.1597/10-145

Nguyen, D. C., Patel, K. B., Skolnick, G. B., Skladman, R., Grames, L. M., Stahl, M. B., Marsh, J. L., \& Woo, A. S. (2015). Progressive Tightening of the Levator Veli Palatini Muscle Improves Velopharyngeal Dysfunction in Early Outcomes of Primary Palatoplasty. Plastic and Reconstructive Surgery, 136(1), 131-141. https://doi.org/10.1097/PRS.0000000000001323

Oliveira, A. C. de A. S. F. de, Scarmagnani, R. H., Fukushiro, A. P., \& Yamashita, R. P. (2016). Influência do treinamento dos avaliadores no julgamento perceptivo da hipernasalidade. CoDAS, 28, 141-148. https://doi.org/10.1590/2317$1782 / 20162015163$

Paal, S., Reulbach, U., Strobel-Schwarthoff, K., Nkenke, E., \& Schuster, M. (2005). Evaluation of Speech Disorders in Children with Cleft Lip and Palate. Journal of Orofacial Orthopedics / Fortschritte Der Kieferorthopädie, 66(4), 270278. https://doi.org/10.1007/s00056-005-0427-2

Padilha, E. Z., Dutka, J. de C. R., Marino, V. C. de C., Lauris, J. R. P., Silva, M. J. F. da, \& Pegoraro-Krook, M. I. (2015). Avaliação da nasalidade de fala na fissura labiopalatina. Audiology - Communication Research, 20, 48-55. https://doi.org/10.1590/S2317-64312015000100001444

Palomares, M., \& Inostroza-Allende, F. (2019). Intervención Fonoaudiológica en pacientes con Fisura Labiopalatina, Insuficiencia velofaríngea y trastornos de habla. En M. P. Moya, F. Susanibar, \& C. Valdés (Eds.), Evaluación e Intervención Logopédica en Motricidad Orofacial y áreas afines ( $1^{\mathrm{a}}$ ed., pp. 201215). Editorial EOS.

Palomares-Aguilera, M., Inostroza-Allende, F., \& Solar, L. R. (2021). Speech pathology telepractice intervention during the COVID-19 pandemic for Spanishspeaking children with cleft palate: A systematic review. International Journal of Pediatric Otorhinolaryngology, 144, 1-10. https://doi.org/10.1016/j.jporl.2021.110700

Pamplona, C., Ysunza, A., Patiño, C., Ramírez, E., Drucker, M., \& Mazón, J. J. (2005). Speech summer camp for treating articulation disorders in cleft palate patients. International Journal of Pediatric Otorhinolaryngology, 69(3), 351-359. https://doi.org/10.1016/j.ijporl.2004.10.012

Paniagua, L. M., Signorini, A. V., Costa, S. S. da, Collares, M. V. M., \& Dornelles, S. (2013). Comparison of videonasoendoscopy and auditory-perceptual evaluation of speech in individuals with cleft lip/palate. International Archives of Otorhinolaryngology, 17(03), 265-273. https://doi.org/10.7162/S180997772013000300006

Rosanowski, F., \& Eysholdt, U. (2002). Phoniatric aspects in cleft lip patients. Facial Plastic Surgery: FPS, 18(3), 197-203. https://doi.org/10.1055/s-200233066

Rullo, R., Di Maggio, D., Festa, V. M., \& Mazzarella, N. (2009). Speech assessment in cleft palate patients: A descriptive study. International Journal of Pediatric Otorhinolaryngology, 73(5), 641-644. https://doi.org/10.1016/j.ijporl.2008.12.011

Scarmagnani, R. H., Barbosa, D. A., Fukushiro, A. P., Salgado, M. H., Trindade, I. E. K., \& Yamashita, R. P. (2015). Relationship between velopharyngeal closure, hypernasality, nasal air emission and nasal rustle in subjects with repaired cleft palate. CoDAS, 27(3), 267-272. https://doi.org/10.1590/2317-1782/20152014145
Sell, D. (2005). Issues in perceptual speech analysis in cleft palate and related disorders: A review. International Journal of Language \& Communication Disorders, 40(2), 103-121. https://doi.org/10.1080/13682820400016522

Sell, D., Grunwell, P., Mildinhall, S., Murphy, T., Cornish, T. A., Bearn, D., Shaw, W. C., Murray, J. J., Williams, A. C., \& Sandy, J. R. (2001). Cleft lip and palate care in the United Kingdom--the Clinical Standards Advisory Group (CSAG) Study. Part 3: Speech outcomes. The Cleft Palate-Craniofacial Journal: Official Publication of the American Cleft Palate-Craniofacial Association, 38(1), 30-37. https://doi.org/10.1597/1545-1569_2001_038_0030_clapci_2.0.co_2

Sell, D., Mildinhall, S., Albery, L., Wills, A. K., Sandy, J. R., \& Ness, A. R. (2015). The Cleft Care UK study. Part 4: Perceptual speech outcomes. Orthodontics \& Craniofacial Research, 18(S2), 36-46. https://doi.org/10.1111/ocr.12112

Sinko, K., Gruber, M., Jagsch, R., Roesner, I., Baumann, A., Wutzl, A., \& DenkLinnert, D.-M. (2017). Assessment of nasalance and nasality in patients with a repaired cleft palate. European Archives of Oto-Rhino-Laryngology, 274(7), 2845-2854. https://doi.org/10.1007/s00405-017-4506-y

Skirko, J. R., Santillana, R. M., Roth, C. T., Dunbar, C., \& Tollefson, T. T. (2018). Spanish Linguistic Validation of the Velopharyngeal Insufficiency Effects on Life Outcomes: VELO-Spanish. Plastic and Reconstructive Surgery - Global Open, 6(11), 1-7. https://doi.org/10.1097/GOX.0000000000001986

Smith, B. E., \& Kuehn, D. P. (2007). Speech evaluation of velopharyngeal dysfunction. The Journal of Craniofacial Surgery, 18(2), 251-261; quiz 266-267. https://doi.org/10.1097/SCS.0b013e31803ecf3b

Smith, B., \& Guyette, T. W. (2004). Evaluation of cleft palate speech. Clinics in Plastic Surgery, 31(2), 251-260. https://doi.org/10.1016/S0094-1298(03)00123-8

Sommerlad, B. C. (2003). A technique for cleft palate repair. Plastic and Reconstructive Surgery, 112(6), 1542-1548. https://doi.org/10.1097/01.PRS.0000085599.84458.D2

Souza, M. T. de, Silva, M. D. da, \& Carvalho, R. de. (2010). Integrative review: What is it? How to do it? Einstein (São Paulo), 8(1), 102-106. https://doi.org/10.1590/s1679-45082010rw1134

Sweeney, T., \& Sell, D. (2008). Relationship between perceptual ratings of nasality and nasometry in children/adolescents with cleft palate and/or velopharyngeal dysfunction. International Journal of Language \& Communication Disorders, 43(3), 265-282. https://doi.org/10.1080/13682820701438177

The jamovi project. (2019). Jamovi-Stats. Open. Now. (1.1) [UNIX]. Jamovi. https://www.jamovi.org/

Tresserra, L., Segovia, J. L., Ballabriga, A., \& Boix, J. (1997). Tratamiento del labio leporino $y$ fisura palatina. JIMS. https://ialnet.unirioja.es/servlet/libro? codigo $=131263$

Trindade, I. E. K., Araújo, B. M. A. M., Teixeira, A. C. M. S., Silva, A. S. C. da, \& Trindade-Suedam, I. K. (2014). Velar activity in individuals with velopharyngeal insufficiency assessed by acoustic rhinometry. Journal of Applied Oral Science, 22(4), 323-330. https://doi.org/10.1590/1678-775720130673

Trost-Cardamone, J. E. (1989). Coming to terms with VPI: A response to Loney and Bloem. The Cleft Palate Journal, 26(1), 68-70. 
Urrútia, G., \& Bonfill, X. (2010). Declaración PRISMA: Una propuesta para mejorar la publicación de revisiones sistemáticas y metaanálisis. Medicina Clínica, 135(11), 507-511. https://doi.org/10.1016/j.medcli.2010.01.015

Whitehill, T., Cheng, J., \& Jones, D. (2007). Rating hypernasality: Direct magnitude (DME) versus visual analogue scaling (VAS). The annual meeting of the American Cleft Palate Craniofacial Association.
Williams, W. N., Bzoch, K. R., Dixon-Wood, V., Seagle, M. B., Nackashi, J. A., Marks, R. G., Frolova, L. E., Serova, E. A., Gonchakov, G. V., Shcheslavskiı̆, S., Shmel'kova, T., \& Zagirova, A. F. (1998). Velopharyngeal function for speech after the Frolova primary palatoplasty technique. The Cleft Palate-Craniofacial Journal: Official Publication of the American Cleft Palate-Craniofacial Association, 35(6), 481-488. https://doi.org/10.1597/1545156919980350481 vffsat 2.3.co 2 